\title{
The Size Distributions of Asteroid Families in the SDSS Moving Object Catalog 4
}

\author{
A. Parker ${ }^{\mathrm{a}, \mathrm{b}} \check{Z}$. Ivezića ${ }^{\mathrm{a}}$ M. Jurićc ${ }^{\mathrm{C}}$ R. Lupton ${ }^{\mathrm{d}}$ M.D. Sekora ${ }^{\mathrm{e}}$ A. Kowalski ${ }^{\mathrm{a}}$ \\ ${ }^{a}$ Department of Astronomy, University of Washington, Seattle, WA 98195, USA \\ ${ }^{\mathrm{b}}$ Department of Astronomy, University of Victoria, Victoria, BC V8W 3P6, Canada \\ ${ }^{\mathrm{c}}$ Institute for Advanced Study, 1 Einstein Drive, Princeton, NJ 08540, USA \\ dPrinceton University Observatory, Princeton, NJ 08544, USA \\ e Applied and Computational Mathematics, Princeton University, Princeton, NJ 08544 , USA
}

\begin{abstract}
Asteroid families, traditionally defined as clusters of objects in orbital parameter space, often have distinctive optical colors. We show that the separation of family members from background interlopers can be improved with the aid of SDSS colors as a qualifier for family membership. Based on an $~ 88,000$ object subset of the Sloan Digital Sky Survey Moving Object Catalog 4 with available proper orbital elements, we define 37 statistically robust asteroid families with at least 100 members (12 families have over 1000 members) using a simple Gaussian distribution model in both orbital and color space. The interloper rejection rate based on colors is typically $\sim 10 \%$ for a given orbital family definition, with four families that can be reliably isolated only with the aid of colors. About $50 \%$ of all objects in this data set belong to families, and this fraction varies from about $35 \%$ for objects brighter than an $H$ magnitude of 13 and rises to $60 \%$ for objects fainter than this. The fraction of C-type objects in families decreases with increasing $H$ magnitude for $H>13$, while the fraction of S-type objects above this limit remains effectively constant. This suggests that S-type objects require a shorter timescale for equilibrating the background and family size distributions
\end{abstract}


via collisional processing. The size distribution varies significantly among families, and is typically different from size distributions for background populations. The size distributions for 15 families display a well-defined change of slope and can be modeled as a "broken" double power-law. Such "broken" size distributions are twice as likely for S-type familes than for C-type families (73\% vs. $36 \%$ ), and are dominated by dynamically old families. The remaining families with size distributions that can be modeled as a single power law are dominated by young families (<1 Gyr). When size distribution requires a double power-law model, the two slopes are correlated and are steeper for S-type families. No such slope-color correlation is discernible for families whose size distribution follows a single power law. For several very populous families, we find that the size distribution varies with the distance from the core in orbital-color space, such that small objects are more prevalent in the family outskirts. This "size sorting" is consistent with predictions based on the Yarkovsky effect.

Keywords: ASTEROIDS; ASTEROIDS, DYNAMICS; PHOTOMETRY 


\section{Introduction}

The size distribution of asteroids is one of most significant observational constraints on their history and is considered to be the "planetary holy grail" (Jedicke \& Metcalfe 1998, and references therein). It is also one of the hardest quantities to determine observationally because of strong selection effects. Recently, Ivezić et al. (2001, hereafter I01) determined the asteroid size distribution to a sub-km limit using multi-color photometry obtained by the Sloan Digital Sky Survey (York et al. 2000; hereafter SDSS). Here we extend their work by using an updated $\left(4^{\text {th }}\right)$ version of the SDSS Moving Object Catalog (Ivezić et al. 2002a, hereafter I02a).

The main goal of this paper is to study size distributions of asteroid families. Asteroid dynamical families are groups of asteroids in orbital element space (Gradie, Chapman \& Williams 1979, Gradie, Chapman \& Tedesco 1989, Valsecchi et al. 1989). This clustering was first discovered by Hirayama (1918, for a review see Binzel 1994), who also proposed that families may be the remnants of parent bodies that broke into fragments. About half of all known asteroids are believed to belong to families; Zappalá et al. 1995 (hereafter Z95), applying a hierarchical clustering method to a sample of 12,487 asteroids, finds over 30 families. Using the same method and a larger sample of 106,000 objects, Nesvorný et al. (2005, hereafter N05) identify 50 statistically robust asteroid families.

The size distributions of asteroid families encode information about their formation and evolution, and constrain the properties of the families' parent bodies (e.g., Marzari, Farinella \& Davis 1999; Tanga et al. 1999; Campo Bagatin \& Petit 2001; Michel et al. 2002; de Elia \& Brunini 2007; Durda

et al. 2007; and references therein). Motivated by this rich information content, as well as the availability of new massive datasets, here we address the following questions

(1) What is the fraction of objects associated with families?

(2) Do objects that are not associated with families show any heliocentric color gradient?

(3) Do objects that are not associated with families have uniform size distribution independent of heliocentric distance? 
(4) Do objects associated with families have a different size distribution than those that are not in families?

(5) Do different families have similar size distributions?

(6) Is the size distribution related to family color and age?

These questions have already been addressed numerous times (e.g. Mikami \& Ishida 1990; Cellino, Zappalá \& Farinella 1991; Marzari, Davis \& Vanzani 1995; Z95; Morbidelli et al. 2003; N05). The main advantages of the size distribution analysis presented here, when compared to previous work, are

- The large sample size: we use a set of $\sim 88,000$ objects for which both SDSS colors and proper orbital elements computed by Milani \& Knežević (1994) are available

- Simple and well-understood selection effects: the SDSS sample is >90\% complete without a strong dependence on magnitude (Jurić et al. 2002, hereafter J02)

- Improved faint limit: the sample of known objects listed in the latest ASTORB le from January 2008 to which SDSS observations are matched is now essentially complete to $r \sim 19.5$ (corresponding to $H \sim 17$ in the inner belt, and to $H \sim 15$ in the outer belt)

- Improved family definitions due to color constraints (rejection of interlopers and separation of families overlapping in orbital space)

- Improved accuracy of absolute magnitudes derived using SDSS photometry, as described below.

The SDSS asteroid data are described in Section 2, and in Section 3 we describe a novel method for defining asteroid families using both orbital parameters and colors. Analysis of the size distribution for families and background objects is presented in Section 4, and we summarize our results in Section 5 . 


\section{SDSS Observations of Moving Objects}

\subsection{An Overview of SDSS}

The SDSS is a digital photometric and spectroscopic survey which will cover about one quarter of the Celestial Sphere in the North Galactic cap, and produce a smaller area $\left(\sim 300 \mathrm{deg}^{2}\right)$ but much deeper survey in the Southern Galactic hemisphere (Stoughton et al. 2002; Abazajian et al. 2003, 2004, 2005; Adelman-McCarthy et al. 2006). SDSS is using a dedicated 2.5m telescope (Gunn et al. 2006) to provide homogeneous and deep $(r<22.5)$ photometry in five bandpasses (Fukugita et al. 1996; Gunn et al. 1998; Smith et al. 2002; Hogg et al. 2001; Tucker et al. 2006) repeatable to 0.02 mag (root-mean-square scatter, hereafter rms, for sources not limited by photon statistics, Ivezić et al. 2003) and with a zeropoint uncertainty of 0.02-0.03 mag (Ivezić et al. 2004). The flux densities of detected objects are measured almost simultaneously in five bands $(u, g, r, i$, and z) with effective wavelengths of $3540 \AA, 4760 \AA, 6280 \AA, 7690 \AA$, and $9250 \AA$. The large survey sky coverage will result in photometric measurements for well over 100 million stars and a similar

number of galaxies 1 . The completeness of SDSS catalogs for point sources is $\sim 99.3 \%$ at the bright end and drops to $95 \%$ at magnitudes of $22.1,22.4,22.1,21.2$, and 20.3 in $u, g, r, i$ and $z$, respectively. Astrometric positions are accurate to better than 0.1 arcsec per coordinate (rms) for sources with $r<20.5$ (Pier et al. 2003), and the morphological information from the images allows reliable stargalaxy separation to $r \sim 21.5$ (Lupton et al. 2002, Scranton et al. 2002). A compendium of other technical details about SDSS can be found on the SDSS web site (http://www.sdss.org), which also provides interface for the public data access.

$\overline{1}$ The recent Data Release 6 lists photometric data for 287 million unique objects observed in 9583 deg $^{2}$ of sky; see http://www.sdss.org/dr6/. 


\subsection{SDSS Moving Object Catalog}

The SDSS, although primarily designed for observations of extragalactic objects, is significantly contributing to studies of the solar system objects because asteroids in the imaging survey must be explicitly detected and measured to avoid contamination of the samples of extragalactic objects selected for spectroscopy. Preliminary analysis of SDSS commissioning data by I01 showed that SDSS will increase the number of asteroids with accurate five-color photometry by more than two orders of magnitude, and to a limit about five magnitudes fainter (seven magnitudes when the completeness limits are compared) than previous multi-color surveys (e.g. The Eight Color Asteroid Survey, Zellner, Tholen \& Tedesco 1985). For example, a comparison of SDSS sample with the Small Main-Belt Asteroid Spectroscopic Survey (Xu et al. 1995; Bus \& Binzel 2002ab) is discussed in detail by $\mathrm{N} 05$.

SDSS Moving Object Catalog 2 (hereafter SDSS MOC) is a public, value-added catalog of SDSS asteroid observations (I02a). It includes all unresolved objects brighter than $r=21.5$ and with observed angular velocity in the $0.05-0.5 \mathrm{deg} /$ day interval. In addition to providing SDSS astrometric and photometric measurements, all observations are matched to known objects listed in the ASTORB file (Bowell 2001), and to a database of proper orbital elements (Milani 1999; Milani \& Knežević 1994)), as described in detail by J02. J02 determined that the catalog completeness (number of moving objects detected by the software that are included in the catalog, divided by the total number of moving objects recorded in the images) is about 95\%, and its contamination rate is about $6 \%$ (the number of entries that are not moving objects, but rather instrumental artifacts). The most recent SDSS MOC 4th data release contains measurements for 471,000 moving objects. A subset of 220,000 observations were matched to 104,000 unique objects listed in the ASTORB file (Bowell 2001). The large sample size increase between the first and fourth release of SDSS MOC is summarized in Figure 1. The object counts in both releases are well described by the following

$\overline{2 \quad \text { http://www.sdss.org/dr6/products/value_added/index.html }}$ 
function (I01)

$$
\frac{\Delta N}{\Delta r}=n(r)=n_{o} \frac{10^{a x}}{10^{b x}+10^{-b x}}
$$

where $x=r-r_{C}, a=\left(k_{1}+k_{2}\right) / 2, b=\left(k_{1}-k_{2}\right) / 2$, with $k_{1}$ and $k_{2}$ the asymptotic slopes of $\log (n)$ vs. $r$ relations. This function smoothly changes its slope around $r \sim r_{C}$, and we find best-fit values $r_{C}=18.5, k_{1}=0.6$ and $k_{2}=0.2$. The normalization constant, $n_{o}$, is 7.1 times larger for SDSS MOC 4 than for the first release. In addition to this sample size increase, the faint completeness limit for objects listed in ASTORB also improved by a about a magnitude, to $r \sim 19.5$ (the number of unique ASTORB objects increased from $\sim 11,000$ to 100,000$)$. Above this completeness limit, the SDSS MOC lists color information for $\sim 33 \%$ of objects listed in ASTORB.

The quality of SDSS MOC data was discussed in detail by I01 and J02, including a determination of the size and color distributions for main-belt asteroids. An analysis of the strong correlation between colors and the main-belt asteroid dynamical families was presented by Ivezić et al. (2002b, hereafter I02b). Jedicke et al. (2004) reported a correlation between the family dynamical age and its mean color for S-type families, and proposed that it is due to space weathering effects. This correlation was further discussed and extended to C-type families by N05. Multiple SDSS observations of objects with known orbital parameters can be accurately linked, and thus SDSS MOC also contains rich information about asteroid color variability, discussed in detail by Szabó et al. (2004) and Szabó and Kiss (2008).

\subsection{Errors in $H$ magnitudes listed in the ASTORB file}

As pointed out by J02, there is a large systematic discrepancy between the absolute magnitudes listed in ASTORB file and values implied by SDSS measurements. The latter are computed as

$$
H_{\text {corr }}=H_{A S T O R B}+V-c V
$$


where $H_{A S T O R B}$ is the ASTORB value, $c V$ is the apparent magnitude in Johnson system computed from information listed in ASTORB as described in J02, and $V$ is the observed magnitude synthesized from SDSS $g$ and $r$ magnitudes (SDSS MOC entries 47, 42, and 32, respectively).

This discrepancy persists in the $4^{\text {th }}$ release of SDSS MOC, as illustrated in Figure 2. The mean difference between $H$ measured by SDSS and the values from ASTORB is 0.23 mag, and the rootmean-scatter is 0.30 mag. The best-fit shown in Figure 2 implies that uncertainty of $H_{\text {corr }}$ is about $0.16 \mathrm{mag}$, with a negligible systematic error (the latter is expected to be about 0.02-0.03 mag due to uncertainties in absolute photometric calibration of SDSS imaging data; see section 2.1). It is likely that this uncertainty is dominated by magnitude variation due to rotation. The magnitude offset of $0.33 \mathrm{mag}$ for $\sim 70 \%$ of measurements implied by the best fit could be due to measurements reported by LINEAR. A similar magnitude offset at the faint end is a known problem in LINEAR calibration, and is currently being addressed with the aid of new calibration catalogs (J.S. Stuart, priv. comm.).

Since the random error in $H$ is twice as large as for $H_{c o r r}$, we adopt $H_{c o r r}$ in the remainder of this work. For a detailed analysis of this magnitude offset problem 3 , we refer the reader to J02.

\section{The Asteroid Families in SDSS MOC}

The contrast between dynamical asteroid families and the background population is especially strong in the space defined by proper orbital elements. These elements are nearly invariants of motion and are thus well suited 4 for discovering objects with common dynamical history (Valsecchi et al. 1989, Milani \& Knežević 1992).

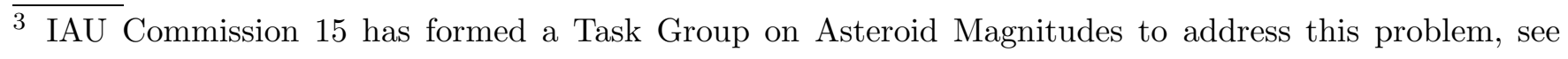
http://www.casleo.gov.ar/c15-wg/index-tgh.html

4 The current asteroid motion is usually described by osculating orbital elements which vary with time due to perturbations caused by planets, and are thus less suitable for studying dynamical families. 
The value of SDSS photometric data becomes particularly evident when exploring the correlation between colors and orbital parameters for main-belt asteroids. I02b demonstrated that asteroid dynamical families, defined as clusters in orbital element space, also strongly segregate in color space. We use the technique developed by I02b to visualize this correlation for $\sim 45,000$ unique mainbelt asteroids with $H_{\text {corr }}<16$ listed in SDSS MOC 4 (Figures 3-4). The asteroid color distribution in SDSS bands shown in Figure 3, and its comparison to traditional taxonomic classifications, is quantitatively discussed by I01 and N05.

A striking feature of Figure 4 is the color homogeneity and distinctiveness displayed by asteroid families. In particular, the three major asteroid families (Eos, Koronis, and Themis), together with the Vesta family, correspond to taxonomic classes K, S, C, and V, respectively (following Burbine et al. 2001, we assume that the Eos family is associated with the $\mathrm{K}$ class). Their distinctive optical colors indicate that the variations in surface chemical composition within a family are much smaller than the compositional differences between families, and vividly demonstrate that asteroids belonging to a particular family have a common origin.

\subsection{A Method for Defining Families Using Orbits and Colors}

Traditionally, the asteroid families are defined as clusters of objects in orbital element space. The most popular methods for cluster definition are the hierarchical clustering and the wavelet analysis (Z95, N05). Given the strong color segregation of families, it is plausible that SDSS colors can be used to improve the orbital family definitions and minimize the mixing of candidate family members and background population.

The SDSS colors used to construct Figures $3-4$ are the $i-z$ color and the so-called $a^{*}$ color, defined in I01 as

$$
a^{*} \equiv 0.89(g-r)+0.45(r-i)-0.57
$$

The $a^{*}$ color is the first principal component of the asteroid color distribution in the SDSS $r-$ 
$i$ vs. $g-r$ color-color diagram (for transformations between the SDSS and Johnson system see Ivezić et al. 2007). Similar principal component analysis was also performed Roig and Gil-Hutton (2006), who considered the distribution of taxonomic classes (especially V-type asteroids) in SDSS principle components by comparing directly to spectroscopic data, and by N05, whose two principal components are well correlated with the $a^{*}$ and $i-z$ colors (we find that $a^{*}=0.49 P C_{1}-0.16$ reproduces the measured $a^{*}$ values with an rms of 0.026 mag for objects with $r<18$ ). The principal colors derived by N05 include the $u$ band, which becomes noisy at the faint end. Given that the completeness of the known object catalog (ASTORB) reaches a faint limit where this noise becomes important, we use the $a^{*}$ and $i-z$ colors to parametrize the asteroid color distribution. Therefore, the family search is performed in a five-dimensional space defined by these two colors and the proper semi-major axis, sine of the inclination angle and eccentricity.

There are numerous techniques that could be used to search for clustering in a multi-dimensional space (e.g. Z95; N05; Carruba \& Michtchenko 2007). They differ in the level of supervision and assumptions about underlying data distribution. Critical assumptions are the distribution shape for each coordinate, their correlations, and the number of independent components. We utilize three different methods, one supervised and two fully automatic. The automatic unsupervised methods are based on the publicly available code FASTMIX 5 by A. Moore and a custom-written code based on Bayesian non-parameteric techniques (Ferguson 1973; Antoniak 1974).

In the supervised method (1) families are manually identified and modeled as orthogonal (i.e. aligned with the coordinate axes) Gaussian distributions in orbital and color space. The two unsupervised methods (2 and 3) also assume Gaussian distributions, but the orientation of individual Gaussians is arbitrary, and the optimal number of families is determined by the code itself. All three methods produce fairly similar results and here we describe only the supervised method (1), and use its results in subsequent analysis. The two unsupervised methods produce generally similar results for the objects associated with families, but tend to overclassify the background into numerous (50-60) small families, and their details and results are not presented thoroughly in this paper.

$\overline{5 \text { See }}$ http://www.cs.cmu.edu/ psand 
We select from the SDSS MOC 4 the first observation of all objects identified in ASTORB, and for which proper orbital elements are also available, resulting in 87,610 objects. Among these, there are 45,502 objects with $H_{\text {corr }}<16$. We split the main sample into three subsets using semimajor axis ranges defined by the major Kirkwood gaps (see Figure 4$)$ : inner $(a<2.50)$, middle $(2.50<a<2.82)$ and outer $(a>2.82)$ belt. For each subset, we produce the $e$ vs. $\sin (i)$ diagrams color-coded analogously to Figure 4, and use them to obtain preliminary identification of asteroid families in both orbital and color space. Approximate rectangular bounds are assigned to these visually identified families, from which median (centroid) and standard deviation, $\sigma$, for the three orbital elements are estimated. Using these estimates, for each asteroid we compute distance in orbital space from a given family centroid as

$$
D_{\text {orbit }}=\sqrt{d_{a}^{2}+d_{e}^{2}+d_{i}^{2}}
$$

where

$$
\begin{aligned}
& d_{a}=\frac{\left(a_{\text {centroid }}-a_{\text {object }}\right)}{\sigma_{a}} \\
& d_{e}=\frac{\left(e_{\text {centroid }}-e_{\text {object }}\right)}{\sigma_{e}} \\
& d_{i}=\frac{\left(i_{\text {centroid }}-i_{\text {object }}\right)}{\sigma_{i}}
\end{aligned}
$$

Histograms in $D_{\text {orbit }}$ were used to determine a preliminary value of $\Delta_{O r b}$, the maximum orbital distance from a family centroid for an object to be ascribed family membership. These initial $\Delta_{\text {Orb }}$ are determined from the differential $D_{\text {orbit }}$ distribution as the position on the first local minimum (the further rise of counts with increasing $D_{\text {orbit }}$ is due to the background objects and other families). The object distribution in the $D_{\text {orbit }}$ vs. $a^{*}$ and $D_{\text {orbit }}$ vs. $(i-z)$ diagrams was used to first define approximate rectangular bounds for each family, and then to compute the color centroid and standard deviation in $a^{*}$ and $(i-z)$ for each candidate family. In order to use color as a family discriminator, we define analogously to orbital elements

$$
D_{\text {color }}=\sqrt{d_{1}^{2}+d_{2}^{2}}
$$


where

$$
\begin{aligned}
& d_{1}=\frac{\left(a_{\text {centroid }}^{*}-a_{\text {object }}^{*}\right)}{\sigma_{a}^{*}} \\
& d_{2}=\frac{\left(i z_{\text {centroid }}-i z_{\text {object }}\right)}{\sigma_{i z}} .
\end{aligned}
$$

We use histograms in $D_{\text {color }}$ to define $\Delta_{C o l}$, the maximum color distance from a family centroid for an object to be ascribed family membership. Figure 8 illustrates the $D_{\text {orbit }}$ and $D_{\text {color }}$ histograms for the Vesta and Baptistina families, and the bottom panels show the distributions of family and background objects in the $D_{\text {orbit }}$ vs. $D_{\text {color }}$ plane.

In cases of families which formed from the disruption of a differentiated parent body, the color distribution might not provide a well defined morphology. However, we did not find any case where a subset of objects selected using $D_{\text {orbit }}$ did not result in one or two well defined color distributions. Nevertheless, it is possible that a small fraction of objects could be rejected from a family due to different colors than the majority of other members.

All objects that have both $D_{\text {orbit }} \leq \Delta_{O r b}$ and $D_{\text {color }} \leq \Delta_{C o l}$ are then considered to be a family member. With a given estimate of family populations, this procedure is iterated and all parameters are refined. It typically takes one to two iterations to converge. All "converged" families are removed from the sample and the process was repeated until there were no family candidates with more than 100 members. This condition is the result of the requirement that the statistical errors for the slope of absolute magnitude distribution of the families are smaller (typically 0.01-0.02) than plausible systematic errors (0.03-0.04), as discussed below.

Using this procedure, we found 37 families which account for $46 \%$ of all objects. Their defining parameters are listed in Table 1. Additional three candidate families that had fewer than 100 members in the last iteration were discarded (see last three entries in Table 2). The family names were determined by comparison with Z95 and N05, and, when no corresponding family was found, by searching for the lowest-numbered asteroid in the Milani \& Knežević (1994) catalog of proper orbital elements. In a small number of cases, it is possible that the "name-giving" object has a color 
that is inconsistent with the majority of objects in the family. We ignored such cases and retained nomenclature from Z95 and N05 in order to ease the comparison. That is, the defining properties of families are the orbital and color parameters listed in Table 1, rather than their names.

The separation of the main-belt asteroids into families and the background objects is illustrated in Figures 5 and 6 . While there is some residual structure in the background, both in color and orbital space, it is much less prominent than the structure displayed by identified families.

\subsection{The Comparison of Resulting Families with Previous Work}

Important questions about the quality of family associations derived here, that are relevant for subsequent analysis, are

- The contamination: are all families robust?

- The completeness: are any families missed?

- What is the impact of variations in adopted family definitions on the resulting family properties such as the number of members and their size distribution?

In this section we address the first two questions, and discuss the third one below (§4.3).

It is unlikely that the derived sample of 37 families contains any spurious family because of the conservative requirement that a family must include at least 100 members. Indeed, we have rejected three familes that are likely real because thay had fewer candidate members (5, 90 and 46). The very narrow color distribution of all selected families provides another argument for their robustness.

Our method of iterative removal of identified families and the simultaneous use of colors and orbital parameters to search for remaining families appears very robust when compared to two automated methods also employed. Nevertheless, given that all three methods from this work assume gaussian distributions of colors and orbital parameters, it is prudent to compare our family list with families obtained by other means, such as the hierarchical clustering method. We have cross-correlated the 
list of 37 families determined here with the list of 41 families obtained by N05 using the hierarchical clustering method. N05 based their study on a larger sample of objects with proper orbital elements ( $\sim 106,000$ vs. $\sim 88,000$ analyzed here; note that the latter sample extends to $\sim 1.5$ mag fainter flux limit but is smaller because it includes only objects observed by SDSS), and did not place a requirement on the minimum number of objects per family. Therefore, it is plausible that families missed by our selected method may be present in their list.

Out of 41 families from the N05 list, 27 are listed in Table 2. This is encouraging level of agreement given the significant difference in applied methodology. We examined in detail each of the fourteen N05 families missing from our list and searched for them in the sample of background objects. We did not find any candidate family that included more than 100 members, though most appear to be real clusters.

Among the ten families from our list that we could not identify in the N05 list, three were detected by at least one method discussed by Z95, and thus are likely real (Euterpe, Teutonia, and Henan). Of the remaining seven, the recognition of four families was greatly aided by color information (Baptistina from Flora, Mitidika and Juno, Lydia and Padua, and McCuskey from Nysa-Polana). It is likely that the remaining three families were not detected by N05 because they have steep absolute magnitude distributions and thus only a small number of members were present in the (older) version of catalog used by N05. For example, among the 3405 objects in the Teutonia family, only 37 have $H_{\text {corr }}<14$.

We further compared our list of families to those presented in Mothe-Diniz et al. (2005), who used spectroscopic measurements to probe asteroid family structure. Of their 21 nominal families, all but three (Renate, Hoffmeister, and Meliboea) can be matched to families detected here. Of the numerous smaller (many with fewer than 100 members) "clumps" they identify, 10 correspond to families listed here, while eight families (Teutonia, Mitidika, Euterpe, Andree, Lydia, Ursula, Lyxaohua and Theobaldia) present in our list do not appear in Mothe-Diniz et al. (2005). They resolved the remaining family in our data set, Flora, into a number of smaller clumps that merged 
into a single family at high cutoff velocities.

A good example of the separation of dynamically mixed families using SDSS colors is provided by the small family Baptistina which is "buried" within the Flora family. Figures 7 and 8 (right panels) illustrate how different $a^{*}$ color distributions enable the identification of $\sim 5 \%$ of the objects nominally assigned Flora family $\left(\left\langle a^{*}\right\rangle=0.13\right)$ membership by their orbital parameters as being members of the Baptistina family $\left(\left\langle a^{*}\right\rangle=-0.04\right)$. While initially puzzled why a similar color-aided search by N05 did not yield any additional families, we have found that all color-separated families extracted here are dominated by faint objects and thus may not have been present in sufficiently large numbers in the older catalog. We conclude that all the families discussed here are robustly detected, and that it is very unlikely that we missed any family with more than 100 hundred members. It is, however, possible that the background is composed of numerous families dominated by small objects that are not discernible with the presently available catalog. Hence, the fraction of $\sim 50 \%$ of objects associated with families is only a lower limit (this fraction is a function of object size, as discussed below).

Figure 11 shows the color dependence of the family and background populations on semi-major axis. We note that the median $a^{*}$ color for the background population becomes bluer as the semi-major axis increases in the same way as the median color for the family population.

\section{The Size Distribution of Asteroid Populations}

The known object catalog is complete to $r \sim 19.5$; above this limit an ASTORB entry is found for practically every SDSS moving object. Depending on the distance and orientation of the observed object, this apparent magnitude limit corresponds to a completenes limit ranging from $H \sim 17$ in the inner belt to $H \sim 15$ in the outer belt. Brighter than these limits, selection function is essentially equal to 1 for the purposes of this work (SDSS managed to observe only about 1/3 of all ASTORB objects, but this is essentially a random selection without an impact on derived absolute magnitude distribution of individual families). These simple selection effects allow us to derive robust family 
size distributions to very small size limits. The only other study of family size distributions that approached the same size limit is that of Morbidelli et al. (2003), who had to introduce an ad hoc indirect correction for selection effects in the known object catalog (which is supported by our analysis, as discussed below).

The transformation between the asteroid absolute magnitude, $H$, and its effective diameter, $D$, requires the knowledge of the absolute visual albedo $p_{V}$,

$$
H=18.1-2.5 \log \left(\frac{p_{V}}{0.1}\right)-5 \log (D / 1 \mathrm{~km})
$$

The absolute albedo is not known for the overwhelming majority of objects in our sample. However, the albedo is known to be strongly correlated with colors (Zellner 1979; Shoemaker et al. 1979; Delbo 2004); for example, the C-like asteroids $\left(a^{*}<0\right)$ have a median albedo of 0.04 and the S-like asteroids have a median albedo of 0.14 . Given that the color variations within a family are small, it seems plausible that the albedo variation within a family is also small (this is supported by the data compiled by Tedesco, Cellino \& Zappalá 2005; see their Table 7). With this assumption, the shapes of the absolute magnitude distribution and the distribution of $\log (D)$ are the same. Hereafter, we will interchangebly use "the absolute magnitude distribution" and "size distribution", where the latter implies the distribution of $\log (D)$. For simplicity, in the remainder of analysis we only use the differential distributions.

If the differential absolute magnitude distribution, $n(H)=\Delta N / \Delta H$, can be described by

$$
\log (n)=\text { Const. }+\alpha H
$$

and the albedos of objects within a given family or population are similar, then it follows from eq. 11 that the differential size distribution can be described as $n(D) \propto D^{-q}$, with the size distribution index

$$
q=5 \alpha+1
$$


While the absolute magnitude distributions derived here often cannot be described by a single power-law, eq. 13 is still useful for locally relating the slope of the $H$ distribution to the slope of implied (differential) $D$ distribution. For example, a model based on an equilibrium cascade in self-similar collisions developed by Dohnanyi (1969) predicts $q=3.5$ and $\alpha=0.5$.

\subsection{The Comparison of Size Distributions for Families and Background}

We compare the size distributions for the family population and for the background, separately for the three regions defined by semi-major axis. The differential absolute magnitude distributions are shown in Figure 9. To aid the comparison of different panels, we plot for reference the differential distribution derived from the cumulative distribution reported by I01

$$
n(r)=n_{o} \frac{10^{a x}}{10^{b x}+10^{-b x}}
$$

where $x=H_{\text {corr }}-H_{C}, a=\left(k_{1}+k_{2}\right) / 2, b=\left(k_{1}-k_{2}\right) / 2$, with $H_{C}=15.5, k_{1}=0.65$ and $k_{2}=0.25$ (Table 4 in I01).

I01 were able to fit this functional form because their sample extended to a $\sim 1.5$ mag fainter $H$ limit $\left(H_{\text {corr }} \sim 17.5\right)$ than the sample discussed here. Given this sample difference, for each $H$ distribution shown in Figure 9 we instead fit a "broken" power law: a separate power-law fit for the bright and faint end. While this procedure is expected to yield a shallower slope at the faint end than the above I01 fit, it is preferred here because it "decouples" the bright and faint ends. The separation of the bright and faint ends was attempted in $H$ steps of $0.5 \mathrm{mag}$, and the value that minimizes the resulting $\chi^{2}$ was adopted as the best fit. The statistical errors for the best-fit slopes are typically $0.01-0.02$, but it is likely that their uncertainty is perhaps a factor of two or so larger due to systematic effects (see $\S 4.3$ below). In a few cases, the best fit is consistent with a single power law. The best-fit power-law parameters for differential absolute magnitude distributions shown in Figure 9 are listed in Table 3. 
The data and best fits shown in the top three panels in Figure 9 demonstrate that the absolute magnitude distributions are not identical: the outer main-belt shows a flatter distribution, and the inner belt shows a steeper distribution than the middle belt region for objects with $H_{\text {corr }}<14$. This is in conflict with the I01 finding that the size distribution appears universal throughout the belt. However, here we analyze a sample about seven times larger; the statistical errors at the bright end for the I01 sample were too large to detect this effect (see their Figures 21 and 22). Nevertheless, the I01 size distribution remains valid when the whole belt is treated together because the counts underprediction of their fit in the outer belt is compensated by its overprediction in the inner belt.

The separation of populations into families and background (the middle and bottom rows in Figure 9) shows that the flattening of $H_{\text {corr }}$ distribution as the semi-major axis increases is valid for each subpopulation separately. Objects associated with families always show the flattening at the faint end, while the background populations admit a single power-law fit in the middle and outer belt.

Due to different $H_{\text {corr }}$ distributions for family and background populations, the fraction of objects associated with families is a function of $H_{\text {corr }}$. The top left panel in Figure 10 shows this dependence separately for blue (dominated by the $\mathrm{C}$ taxonomic type) and red (dominated by the $\mathrm{S}$ type) subsets. For both subsets, the fraction of objects in families significantly increases from $\sim 20 \%$ to $\sim 50 \%$ between $H_{\text {corr }}=9$ and $H_{\text {corr }}=11$. The two color-selected subsets show different behavior for $H_{\text {corr }}>11$ : for blue subset the fraction of objects in families decreases from $\sim 50 \%$ to $\sim 30 \%$, while it stays constant at the $\sim 60 \%$ level for red families. Since blue families typically have larger semi-major axis than red families, it is possible that this decrease in family membership is due to increasing color rejection at the faint end. However, the remaining two panels in Figure 10 demonstrate that this is not the case because the color rejection rate is both fairly independent of $H_{\text {corr }}$, and too small to account for the observed decrease of blue family membership.

The dominance of background objects for $H_{c o r r}<13$ is consistent with the background population having a significantly shallower size distribution for large objects than the families. The falloff of blue 
family fraction and the slow climb in color rejection rate toward large values of $H_{\text {corr }}$ in Figure 10 confirms that the size distributions for blue families are shallower for values of $H_{\text {corr }}>13$. Because the red family fraction is effectively flat to our detection limit it appears that the red family and background populations have identical size distributions for objects with $H_{\text {corr }}>13$. Morbidelli et al (2003) suggest that the background population is composed of many small families which formed from small-diameter objects and as such should have steep size distributions for objects larger than 1 $\mathrm{km}$, producing a background population with an initial size distribution (for objects $>1 \mathrm{~km}$ ) steeper than that for families which formed from the breakup of larger objects. Differences between the size distributions of these two populations should eventually disappear through collisional processing. Because the blue family and background populations appear to have significantly different size distributions while the red populations' size distributions appear identical (for $H_{\text {corr }}>13$ ), we infer that the time for equilibrating the background and family size distributions is longer for blue objects than red. This difference in the equilibration time may be due either to differences in asteroid internal structure and material properties between taxonomic classes or due to environmental variations in collisional processing rates, as red objects are more prevalent in the inner belt and blue objects in the outer belt.

We note that the background population in the outer belt shows a curious excess of large objects $\left(H_{\text {corr }}<11.5\right)$ compared to best power-law fit (Figure 9, bottom right). We have inspected the orbital parameter and color distributions for 58 objects with $10<H_{\text {corr }}<11$ and found that they are not associated with any identified family, nor generally clustered.

\subsection{The Comparison of Size Distributions for Individual Families}

The inspection of differential $H_{\text {corr }}$ distributions for the 37 families identified here shows that many, but not all, display a clear change of slope such as seen for family populations in Figure 9 . We have attempted a "broken" power-law fit for all families. When the two best-fit slopes differ by less than 0.05, we enforce a single power-law fit. This procedure yields 22 families described by a single power 
law and 15 families with a robust detection of the slope change. Their best-fit parameters are listed in Tables 4 and 5, respectively, and a few examples of measured $H$ distributions and best fits are shown in Figure 12.

For families whose absolute magnitude distributions are described by a single power law, the median best-fit power-law slope is 0.56 , with a standard deviation of 0.16 (determined form inter-quartile range). This scatter is significantly larger than the measurement errors and indicate that families do not have a universal size distribution. Similarly, for families with a best-fit "broken" power law, the medians and standard deviations for the "bright" and "faint" slopes are $(0.66,0.24)$ and $(0.32$, 0.15), respectively (again note the significant scatter relative to the measurement errors), with the median $H_{\text {corr }}$ where the slope changes of $14.2\left(D \sim 6 \mathrm{~km}\right.$ for $\left.p_{V}=0.1\right)$. We discuss correlations of these best-fit parameters with the family color and age in $§ 4.4$.

\subsection{Systematic Deviations in Size Distribution due to Variations in Family Definitions}

Before proceeding with the analysis of correlations between size distributions and other family properties such as color and age, we analyze the systematic deviations in size distribution due to variations in family definitions. For example, the color constraints may result in a size-dependent incompleteness because of the increased photometric noise at the faint end. Similarly, the assumption of gaussian distributions for orbital parameters and colors may result in incomplete families due to extended halos, as pointed out by N05. This effect may also induce size-dependant systematics because small objects are scattered over a larger region of orbital space, as shown below.

The Vesta family offers a good test case because of its unique color distribution (which is due to the influence of $1 \mu \mathrm{m}$ absorption feature on the measured $i-z$ color). The top panel in Figure 13 compares the $H_{c o r r}$ distributions for the adopted Vesta family and for a less constraining orbital cut defined simply by $0.06<\sin (i)<0.16$ and $e<0.16$, that yields $30 \%$ more candidate members. Apart from this overall shift in the normalization, the resulting distributions have statistically indis-

tinguishable shapes. The middle panel compares the adopted family and a much less constraining 
color cut, $a^{*}>0$ (i.e. no constraint on the $i-z$ color), that yields $50 \%$ more objects. Again, the slope of the two distributions are indistinguishable.

We detect a significant difference, however, when we split the adopted family in the "core" and "outskirt" parts using $D_{\text {orbit }}<1$ and $1.75<D_{\text {orbit }}<2.75$ (see eq. 4). As the bottom panel in Figure 13 shows, the "outskirt" subsample has a steeper $H_{\text {corr }}$ distribution than the "core" subsample. The best-fit power-law slopes in the $14<H_{\text {corr }}<16$ region are 0.45 and 0.59 for the "core" and "outskirt" subsample, respectively. We note that despite this slope difference, the change of slope between the bright (0.89) and faint end is robustly detected.

Another method to see the same "size sorting" effect is to inspect the dependence of $D_{\text {orbit }}$ on $H_{\text {corr }}$. We find that the median $D_{\text {orbit }}$ for objects in the Vesta family increases from 1.0 to 1.5 as $H_{\text {corr }}$ increases from 14 to 17.

This "size sorting" effect is not a peculiar property of the Vesta family as it is seen for a large fraction of families. It is caused by an increased scatter in all three orbital parameters as $H_{\text {corr }}$ increases. This is not surprising as the velocity field of the fragments produced in the disruption of an asteroid family's parent body may have been size-dependent. For most families the sorting is dominated by the increased dispersion in the semi-major axis. One of the most striking examples, the Eos family, is shown in Figure 14. As discussed by N05, this increase of dispersion as size decreases can be also be explained as the drift induced by the Yarkovsky effect (see also Vokrouhlický 1999; and Bottke et al. 2001).

\subsection{Correlations between Size Distributions and Family Color and Age}

We analyze the correlations between the best-fit size distribution parameters listed in Tables 4 and 5, and family color and age. The age, when available, is taken from the compilation by N05.

The dependence of the power-law index on the mean $a^{*}$ color for families described by a single power law is shown in the top left panel in Figure 15. The mean and standard deviation for 14 blue 
families are $(0.55,0.13)$, and for 8 red families are $(0.65,0.19)$. These differences are not statistically significant. Within each color-selected subsample (blue vs. red, i.e. $a^{*}<0$ vs $a^{*}>0$ ), there is no discernible correlation between the slope and color.

Families that require a "broken" power law fit are twice as likely for red families $\left(a^{*}>0\right)$ dominated by $\mathrm{S}$ type asteroids) than for blue families dominated by $\mathrm{C}$ type asteroids (73\% vs. 36\%). As illustrated in Figure 15, the size distributions are systematically steeper for S type families, and the "bright" and "faint" end slopes appear to be correlated. The median values of the "bright" and "faint" end slopes are $(0.57,0.18)$ for blue families, and $(0.79,0.39)$ for red families.

For a subset of families that have available age estimates, we find that families with "broken" power law size distributions are dominated by old families, while those that admit a single power law are dominated by young families, with the age separation boundary at $\sim 1$ Gyr. We note that the size distribution was used for some of age estimates compiled by N05, so this conclusion may be a bit of circular reasoning, though the majority of age estimates are derived independently of the observed size distribution.

The correlations between the mean color and family age reported by Jedicke et al. (2004) and N05 are reproduced when using the $a^{*}$ color for families discussed here. Figure 16 illustrates a good agreement with the analytic fits to the observed correlations obtained by N05, and further demonstrates the correlation between the observed size distributions and age.

\section{Discussion and Conclusions}

We have used a large sample of asteroids $(\sim 88,000)$ for which both orbital elements and SDSS colors are available to derive improved membership for 37 asteroid families. The addition of colors typically rejects about $10 \%$ of all dynamically identified candidate members due to mismatched colors. Four families can be reliably isolated only with the aid of colors. About $50 \%$ of objects in this data set belong to families, with this fraction representing a lower limit due to a conservative requirement 
that a candidate family must include at least 100 members. The resulting family definitions are in good agreement with previous work (e.g. Z95, N05) and all the discrepancies are well understood. Although SDSS has observed only about $1 / 3$ of all known asteroids, it is remarkable that the sample discussed here provides color information for more than an order of magnitude more objects associated with families than analyzed in the published literature.

This data set enables the determination of absolute magnitude (size) distributions for individual families to a very faint limit without a need to account for complex selection effects. We verify that size distribution varies significantly among families, and is typically different from size distributions for background populations. Consequently, the asteroid size distribution cannot be described by a universal function that is valid throughout the main belt (e.g. Jedicke \& Metcalfe 1998, Ivezić et al. 2001, and reference therein). This finding will have an influence on conclusions derived from modeling the size distribution under this assumption (e.g. Bottke et al. 2005, and references therein). In particular, it is not clear how to interpret a detailed dependence of the critical specific energy (energy per unit mass required to fragment an asteroid and disperse the fragments to infinity) on asteroid size derived from such models, when the starting observational constraint on size distribution is an average over multiple families with significantly varying size distributions.

We show that for objects with $H_{\text {corr }}<13$, the background population dominates (family fraction decreases toward lower $H_{c o r r}$, indicating a shallower size distribution for large objects), while for objects with $H_{\text {corr }}>13$, the red family fraction remains effectively constant to our completeness limit while the blue family fraction falls off. This indicates that the time to collisionally equilibrate the family and background populations (see e.g., Morbidelli et al. 2003) is shorter for red objects than blue.

The size distributions for 15 families display a well-defined change of slope and can be modeled as a "broken" double power-law. The first evidence for this effect and a discussion of its significance are presented by Morbidelli et al. (2003). Using a data set with much simpler correction for the observational selection effects, we confirm their result in a statistically more robust way. We also 
find such "broken" size distributions are twice as likely for S-type familes than for C-type families (73\% vs. 36\%), and are dominated by dynamically old families. The remaining families with size distributions that can be modeled as a single power law are dominated by young families $(<1$ Gyr).

The eight largest families all show a change of size distribution slope to much smaller values at the faint end (see Table 5). This result has a direct consequence when prediciting the number of very small objects $(D \sim 1 \mathrm{~km})$. In particular, it could explain why the Statistical Asteroid Model developed by Tedesco, Cellino \& Zappalá (2005) predicts too many objects: the data presented here are inconsistent with the SAM assumptions for the number of objects in its most populous families such as Eunomia and Themis.

We find that when size distribution requires a double power-law model, the two slopes are correlated and are steeper for S-type families. No such slope-color correlation is discernible for families whose size distribution follows a single power law. While beyond the scope of this work, the modeling of such correlations may shed light on the internal structure and material properties of asteroids.

For several very populous families, we find that the size distribution varies with the distance from the core in orbital-color space, such that small objects are more prevalent in the family outskirts. As discussed by N05 (and references therein), this "size sorting" is consistent with predictions based on the Yarkovsky/YORP effect.

While these results provide significant new observational constraints for the properties of main-belt asteroids, very soon the observations will further improve. The upcoming large-scale sky surveys, such as Pan-STARRS (Kaiser et al. 2002) and LSST (Tyson 2002), will obtain even more impressive samples, both in size, diversity of measurements and their accuracy. For example, LSST will scan the whole observable sky every three nights in two bands to a $5 \sigma$ depth equivalent to $V=24.7$. These data will enable much improved analysis due to several factors

- Due to hundreds of observations, the orbits will be determined directly, instead of relying on external data, resulting in a sample about 30-40 times larger than discussed here

- The effective faint limit will be extended by about 5 magnitudes, correspoding to ten times smaller 
size limit (diameters of several hundred meters)

- Due to many photometric observations obtained with the same well-calibrated system, the uncertainties in absolute magnitudes will be an order of magnitude smaller

- The addition of the $y$ band (at $\sim 1 \mu \mathrm{m}$ ) will improve the color classification due to better sensitivity to the $\sim 1 \mu \mathrm{m}$ absorption feature present in spectra of many asteroids.

These new data will undoubtely reinvigorate both observational and theoretical studies of main-belt asteroids.

\section{Acknowledgments}

We are grateful to E. Bowell for making his ASTORB file publicly available, and to A. Milani, Z. Knežević and their collaborators for generating and distributing proper orbital elements. M.J. gratefully acknowledges support from the Taplin Fellowship and from NSF grant PHY-0503584.

Funding for the SDSS and SDSS-II has been provided by the Alfred P. Sloan Foundation, the Participating Institutions, the National Science Foundation, the U.S. Department of Energy, the National Aeronautics and Space Administration, the Japanese Monbukagakusho, the Max Planck Society, and the Higher Education Funding Council for England. The SDSS Web Site is http://www.sdss.org/.

The SDSS is managed by the Astrophysical Research Consortium for the Participating Institutions. The Participating Institutions are the American Museum of Natural History, Astrophysical Institute Potsdam, University of Basel, University of Cambridge, Case Western Reserve University, University of Chicago, Drexel University, Fermilab, the Institute for Advanced Study, the Japan Participation Group, Johns Hopkins University, the Joint Institute for Nuclear Astrophysics, the Kavli Institute for Particle Astrophysics and Cosmology, the Korean Scientist Group, the Chinese Academy of Sciences (LAMOST), Los Alamos National Laboratory, the Max-Planck-Institute for Astronomy (MPIA), the Max-Planck-Institute for Astrophysics (MPA), New Mexico State University, Ohio State University, University of Pittsburgh, University of Portsmouth, Princeton University, the 
United States Naval Observatory, and the University of Washington.

\section{References}

Abazajian, K., and 109 coauthors 2004. The Second Data Release of the Sloan Digital Sky Survey. Astron. J. 128, 502-512.

Abazajian, K., and 109 coauthors 2005. The Third Data Release of the Sloan Digital Sky Survey. Astron. J. 129, 1755-1759.

Abazajian, K., and 135 coauthors 2003. The First Data Release of the Sloan Digital Sky Survey. Astron. J. 126, 2081-2086.

Adelman-McCarthy, J. K., and 100 coauthors 2006. The Fourth Data Release of the Sloan Digital Sky Survey. Astrophys. J. Suppl. Ser. 162, 38-48.

Antoniak, C. 1974. Mixtures of dirichlet processes with applications to bayesian nonparametric problems. The Annals of Statistics 2(1152).

Binzel, R. P. 1994. Physical Studies of Hirayama Families: Recent Results and Future Prospects (invited). In Y. Kozai, R. P. Binzel, and T. Hirayama (Eds.), 75 Years of Hirayama Asteroid Families: The Role of Collisions in the Solar System History, Volume 63 of Astronomical Society of the Pacific Conference Series, pp. 251-+.

Bottke, W. F., D. D. Durda, D. Nesvorný, R. Jedicke, A. Morbidelli, D. Vokrouhlický, and H. F. Levison 2005. Linking the collisional history of the main asteroid belt to its dynamical excitation and depletion. Icarus 179, 63-94.

Bottke, W. F., D. Vokrouhlický, M. Broz, D. Nesvorný, and A. Morbidelli 2001. Dynamical Spreading of Asteroid Families by the Yarkovsky Effect. Science 294, 1693-1696.

Bowell, E. 2001. The asteroid orbital elements database. (ASTORB, Flagstaff: Lowell Obs.).

Burbine, T. H., R. P. Binzel, S. J. Bus, and B. E. Clark 2001. K asteroids and CO3/CV3 chondrites. Meteoritics and Planetary Science 36, 245-253.

Bus, S. J., and R. P. Binzel 2002a. Phase II of the Small Main-Belt Asteroid Spectroscopic SurveyA Feature-Based Taxonomy. Icarus 158, 146-177. 
Bus, S. J., and R. P. Binzel 2002b. Phase II of the Small Main-Belt Asteroid Spectroscopic SurveyThe Observations. Icarus 158, 106-145.

Campo Bagatin, A., and J.-M. Petit 2001. Effects of the Geometric Constraints on the Size Distributions of Debris in Asteroidal Fragmentation. Icarus 149, 210-221.

Carruba, V., and T. A. Michtchenko 2007. A frequency approach to identifying asteroid families. Astron. Astrophys. 475, 1145-1158.

Cellino, A., V. Zappala, and P. Farinella 1991. The size distribution of main-belt asteroids from IRAS data. Mon. Not. R. Astron. Soc. 253, 561-574.

de Elía, G. C., and A. Brunini 2007. Collisional and dynamical evolution of the main belt and NEA population. Astron. Astrophys. 466, 1159-1177.

Delbo, M. 2004. The nature of near-earth asteroids from the study of their thermal infrared emission. Ph. D. thesis, Freie Universität, Berlin.

Dohnanyi, J. W. 1969. Collisional models of asteroids and their debris. J. Geophys. Res. 74, $2531-2554$.

Durda, D. D., W. F. Bottke, D. Nesvorný, B. L. Enke, W. J. Merline, E. Asphaug, and D. C. Richardson 2007. Size frequency distributions of fragments from SPH/N-body simulations of asteroid impacts: Comparison with observed asteroid families. Icarus 186, 498-516.

Ferguson, T. 1973. A bayesian analysis of some nonparametric problems. The Annals of Statistics $1((2)), 209-230$.

Fukugita, M., T. Ichikawa, J. E. Gunn, M. Doi, K. Shimasaku, and D. P. Schneider 1996. The Sloan Digital Sky Survey Photometric System. Astron. J. 111, 1748-+.

Gradie, J. C., C. R. Chapman, and E. F. Tedesco 1989. Distribution of taxonomic classes and the compositional structure of the asteroid belt. In R. P. Binzel, T. Gehrels, and M. S. Matthews (Eds.), Asteroids II, pp. 316-335.

Gradie, J. C., C. R. Chapman, and J. G. Williams 1979. Families of minor planets, pp. 359-390. Asteroids.

Gunn, J. E., and 29 coauthors 1998. The Sloan Digital Sky Survey Photometric Camera. Astron. J. 116, 3040-3081. 
Gunn, J. E., and 42 coauthors 2006. The 2.5 m Telescope of the Sloan Digital Sky Survey. Astron. J. 131, 2332-2359.

Hirayama, K. 1918. Groups of asteroids probably of common origin. Astron. J. 31, 185-188.

Hogg, D. W., D. P. Finkbeiner, D. J. Schlegel, and J. E. Gunn 2001. A Photometricity and Extinction Monitor at the Apache Point Observatory. Astron. J. 122, 2129-2138.

Ivezić, Ž., and 10 coauthors 2003. Variability Studies with SDSS. Memorie della Societa Astronomica Italiana $74,978-+$.

Ivezić, Ž., and 19 coauthors 2004. SDSS data management and photometric quality assessment. Astronomische Nachrichten 325, 583-589.

Ivezić, Ž., and 22 coauthors 2007. Sloan Digital Sky Survey Standard Star Catalog for Stripe 82: The Dawn of Industrial 1\% Optical Photometry. Astron. J. 134, 973-998.

Ivezić, Ž., 23 coauthors, and the SDSS Collaboration 2001. Solar System Objects Observed in the Sloan Digital Sky Survey Commissioning Data. Astron. J. 122, 2749-2784.

Ivezić, Ž., M. Juric, R. H. Lupton, S. Tabachnik, and T. Quinn 2002. Asteroids Observed by The Sloan Digital Survey. In J. A. Tyson and S. Wolff (Eds.), Survey and Other Telescope Technologies and Discoveries. Edited by Tyson, J. Anthony; Wolff, Sidney. Proceedings of the SPIE, Volume 4836, pp. 98-103 (2002)., Volume 4836 of Presented at the Society of Photo-Optical Instrumentation Engineers (SPIE) Conference, pp. 98-103.

Ivezić, Ž., R. H. Lupton, M. Jurić, S. Tabachnik, T. Quinn, J. E. Gunn, G. R. Knapp, C. M. Rockosi, and J. Brinkmann 2002. Color Confirmation of Asteroid Families. Astron. J. 124, 2943-2948.

Jedicke, R., and T. S. Metcalfe 1998. The Orbital and Absolute Magnitude Distributions of Main Belt Asteroids. Icarus 131, 245-260.

Jedicke, R., D. Nesvorný, R. Whiteley, Ž. Ivezić, and M. Jurić 2004. An age-colour relationship for main-belt S-complex asteroids. Nature 429, 275-277.

Jurić, M., and 11 coauthors 2002. Comparison of Positions and Magnitudes of Asteroids Observed in the Sloan Digital Sky Survey with Those Predicted for Known Asteroids. Astron. J. 124, $1776-1787$.

Kaiser, N., and 19 coauthors 2002. Pan-STARRS: A Large Synoptic Survey Telescope Array. 
In J. A. Tyson and S. Wolff (Eds.), Survey and Other Telescope Technologies and Discoveries. Edited by Tyson, J. Anthony; Wolff, Sidney. Proceedings of the SPIE, Volume 4836, pp. 154-164 (2002)., Volume 4836 of Presented at the Society of Photo-Optical Instrumentation Engineers (SPIE) Conference, pp. 154-164.

Lupton, R. H., Z. Ivezic, J. E. Gunn, G. Knapp, M. A. Strauss, and N. Yasuda 2002. SDSS Imaging Pipelines. In J. A. Tyson and S. Wolff (Eds.), Survey and Other Telescope Technologies and Discoveries. Edited by Tyson, J. Anthony; Wolff, Sidney. Proceedings of the SPIE, Volume 4836, pp. 350-356 (2002)., Volume 4836 of Presented at the Society of Photo-Optical Instrumentation Engineers (SPIE) Conference, pp. 350-356.

Marzari, F., D. Davis, and V. Vanzani 1995. Collisional evolution of asteroid families. Icarus 113, 168-187.

Marzari, F., P. Farinella, and D. R. Davis 1999. Origin, Aging, and Death of Asteroid Families. Icarus $142,63-77$.

Michel, P., P. Tanga, W. Benz, and D. C. Richardson 2002. Formation of Asteroid Families by Catastrophic Disruption: Simulations with Fragmentation and Gravitational Reaccumulation. Icarus 160, 10-23.

Mikami, T., and K. Ishida 1990. Size distributions of member asteroids in seven Hirayama families. Pub. Astron. Soc. Japan 42, 165-174.

Milani, A. 1999. The Asteroid Identification Problem. I. Recovery of Lost Asteroids. Icarus 137, 269-292.

Milani, A., and Z. Knežević 1992. Asteroid proper elements and secular resonances. Icarus 98, $211-232$.

Milani, A., and Z. Knežević 1994. Asteroid proper elements and the dynamical structure of the asteroid main belt. Icarus 107, 219-254.

Morbidelli, A., D. Nesvorný, W. F. Bottke, P. Michel, D. Vokrouhlický, and P. Tanga 2003. The shallow magnitude distribution of asteroid families. Icarus 162, 328-336.

Mothé-Diniz, T., F. Roig, and J. M. Carvano 2005. Reanalysis of asteroid families structure through visible spectroscopy. Icarus $\mathbf{1 7 4}, 54-80$. 
Nesvorný, D., R. Jedicke, R. J. Whiteley, and Ž. Ivezić 2005. Evidence for asteroid space weathering from the Sloan Digital Sky Survey. Icarus 173, 132-152.

Pier, J. R., J. A. Munn, R. B. Hindsley, G. S. Hennessy, S. M. Kent, R. H. Lupton, and Ž. Ivezić 2003. Astrometric Calibration of the Sloan Digital Sky Survey. Astron. J. 125, 1559-1579.

Roig, F., and R. Gil-Hutton 2006. Selecting candidate V-type asteroids from the analysis of the Sloan Digital Sky Survey colors. Icarus 183, 411-419.

Scranton, R., and 33 coauthors 2002. Analysis of Systematic Effects and Statistical Uncertainties in Angular Clustering of Galaxies from Early Sloan Digital Sky Survey Data. Astrophys. J. 579, 48-75.

Shoemaker, E. M., J. G. Williams, E. F. Helin, and R. F. Wolfe 1979. Earth-crossing asteroids Orbital classes, collision rates with earth, and origin, pp. 253-282. Asteroids.

Smith, J. A., and 16 coauthors 2002. The u'g'r'i'z' Standard-Star System. Astron. J. 123, 21212144.

Stoughton, C., and 140 coauthors 2002. Sloan Digital Sky Survey: Early Data Release. Astron. J. 123, 485-548.

Szabó, G. M., Ž. Ivezić, M. Jurić, R. Lupton, and L. L. Kiss 2004. Colour variability of asteroids in the Sloan Digital Sky Survey Moving Object Catalog. Mon. Not. R. Astron. Soc. 348, 987-998.

Szabó, G. M., and L. L. Kiss 2008. The shape distribution of asteroid families - evidence for evolution driven by small impacts. Icarus, in press 801.

Tanga, P., A. Cellino, P. Michel, V. Zappalà, P. Paolicchi, and A. dell'Oro 1999. On the Size Distribution of Asteroid Families: The Role of Geometry. Icarus 141, 65-78.

Tedesco, E. F., A. Cellino, and V. Zappalá 2005. The Statistical Asteroid Model. I. The Main-Belt Population for Diameters Greater than 1 Kilometer. Astron. J. 129, 2869-2886.

Tucker, D. L., and 24 coauthors 2006. The Sloan Digital Sky Survey monitor telescope pipeline. Astronomische Nachrichten 327, 821-+.

Tyson, J. A. 2002. Large Synoptic Survey Telescope: Overview. In J. A. Tyson and S. Wolff (Eds.), Survey and Other Telescope Technologies and Discoveries. Edited by Tyson, J. Anthony; Wolff, Sidney. Proceedings of the SPIE, Volume 4836, pp. 10-20 (2002)., Volume 4836 of Presented at 
the Society of Photo-Optical Instrumentation Engineers (SPIE) Conference, pp. 10-20.

Valsecchi, G. B., A. Carusi, Z. Knežević, L. Kresak, and J. G. Williams 1989. Identification of asteroid dynamical families. In R. P. Binzel, T. Gehrels, and M. S. Matthews (Eds.), Asteroids II, pp. 368-385.

Vokrouhlický, D. 1999. A complete linear model for the Yarkovsky thermal force on spherical asteroid fragments. Astron. Astrophys. 344, 362-366.

Xu, S., R. P. Binzel, T. H. Burbine, and S. J. Bus 1995. Small main-belt asteroid spectroscopic survey: Initial results. Icarus 115, 1-35.

York, D. G., and 105 coauthors 2000. The Sloan Digital Sky Survey: Technical Summary. Astron. J. 120, 1579-1587.

Zappala, V., P. Bendjoya, A. Cellino, P. Farinella, and C. Froeschle 1995. Asteroid families: Search of a 12,487-asteroid sample using two different clustering techniques. Icarus 116, 291-314.

Zellner, B. 1979. Asteroid taxonomy and the distribution of the compositional types, pp. 783-806. Asteroids.

Zellner, B., D. J. Tholen, and E. F. Tedesco 1985. The eight-color asteroid survey - Results for 589 minor planets. Icarus $\mathbf{6 1}, 355-416$. 


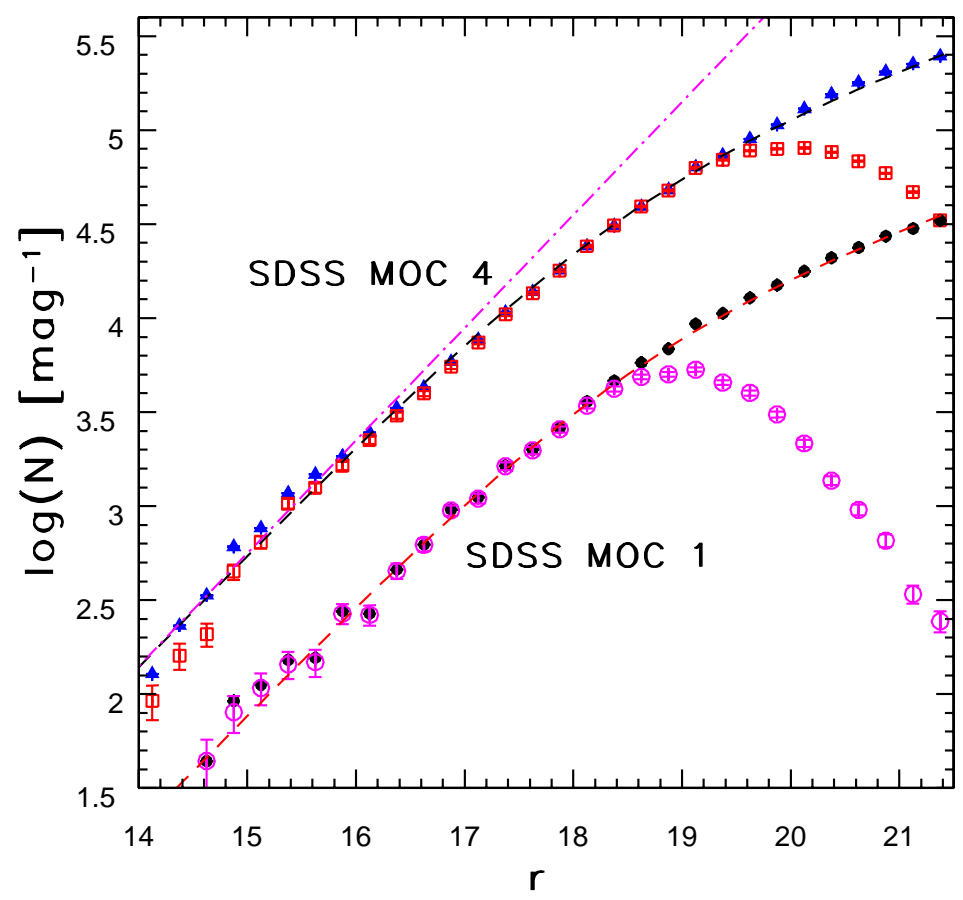

Fig. 1. An illustration of the improvements in sample size between the first and the fourth release of SDSS Moving Object Catalog. Symbols with (statistical) error bars show differential counts for moving objects listed in the first release from 2002 (dots: all objects detected by SDSS; circles: identified in ASTORB file) and the fourth release from 2008 (triangles: all SDSS; squares: in ASTORB). Note that, in addition to a sample size increase of about a factor of 7 , the faint completeness limit for objects listed in ASTORB also improved by a about a magnitude (the number of unique ASTORB objects increased from 11,000 to $\sim 100,000)$. The dashed lines show a double-power law fit described in text, with the $d \log (N) / d r$ slope changing from 0.60 at the bright end to 0.20 at the faint end. For illustration, the dot-dashed lines shows a single power-law with a slope of 0.60 . 


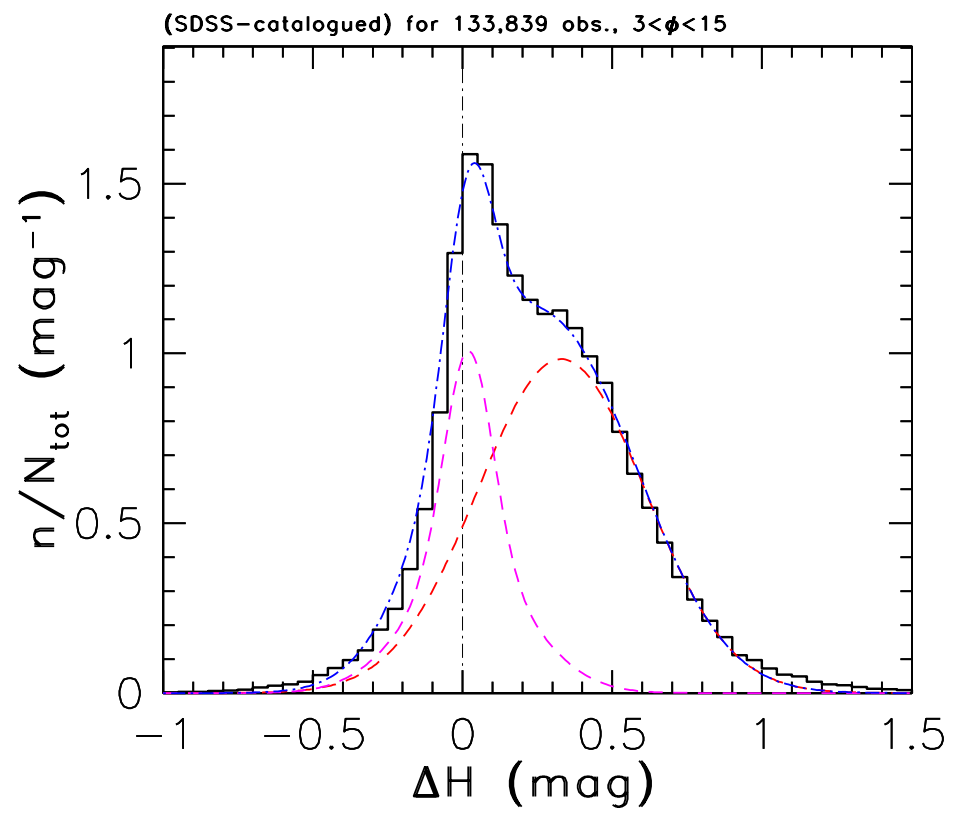

Fig. 2. A comparison of asteroid absolute magnitude, $H$, inferred from SDSS measurements, and the value listed in ASTORB file for 133,000 observations of about 64,000 unique objects observed at phase angles between 3 and 15 degrees. The histogram shows the data distribution $\left(\Delta H=H_{S D S S}-H_{A S T O R B}=H_{c o r r}-H\right.$, see eq. 2) and the dot-dashed line is a best fit. The best fit is a linear combination of three gaussians: two (which simulate asteroid variability) are centered on 0.02 , have widths of 0.08 and 0.20 mag, and have relative normalizations of $13 \%$ and $18 \%$, respectively. Their sum is shown by the dashed line centered on $\Delta H=0.02$. The third gaussian (which accounts for a large number of objects with bad photometry) has a width of $0.28 \mathrm{mag}$ and is shown by the dashed line centered on $\Delta H=0.33$. That is, about $69 \%$ of $H$ measurements listed in ASTORB file are systematically too bright by 0.33 mag. 


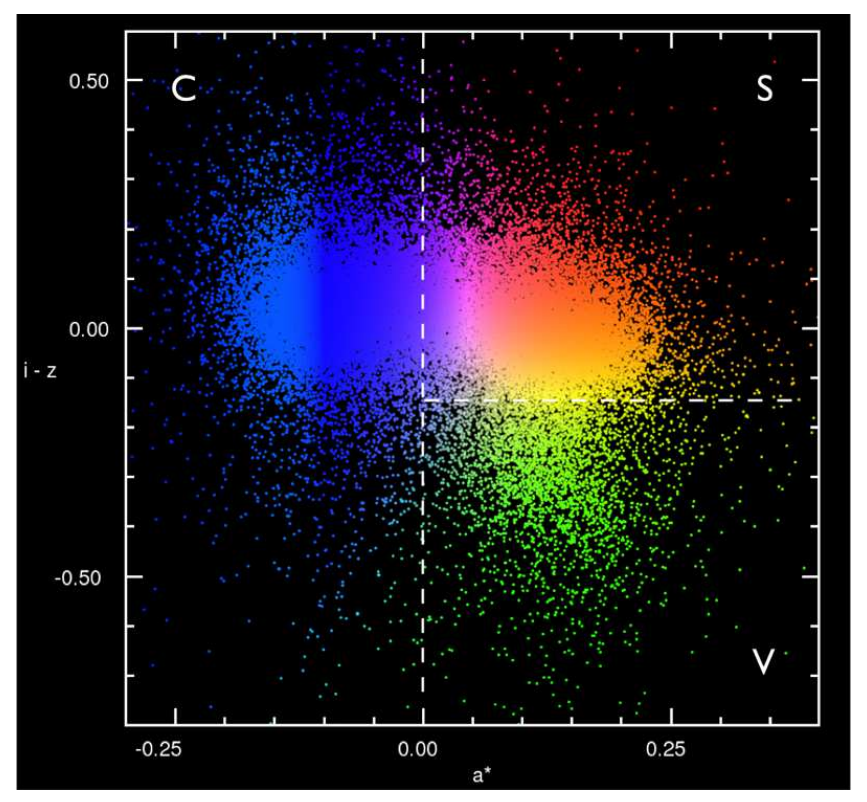

Fig. 3. A plot of the color distribution in $a *$ and $i-z$ of 45,087 unique objects listed in both the SDSS MOC 4 and ASTORB file, and that have $H_{c o r r}<16$. The approximate boundaries of three spectral classes are marked, and used in labeling family type. The color-coding scheme defined here is used in figures 4-6.

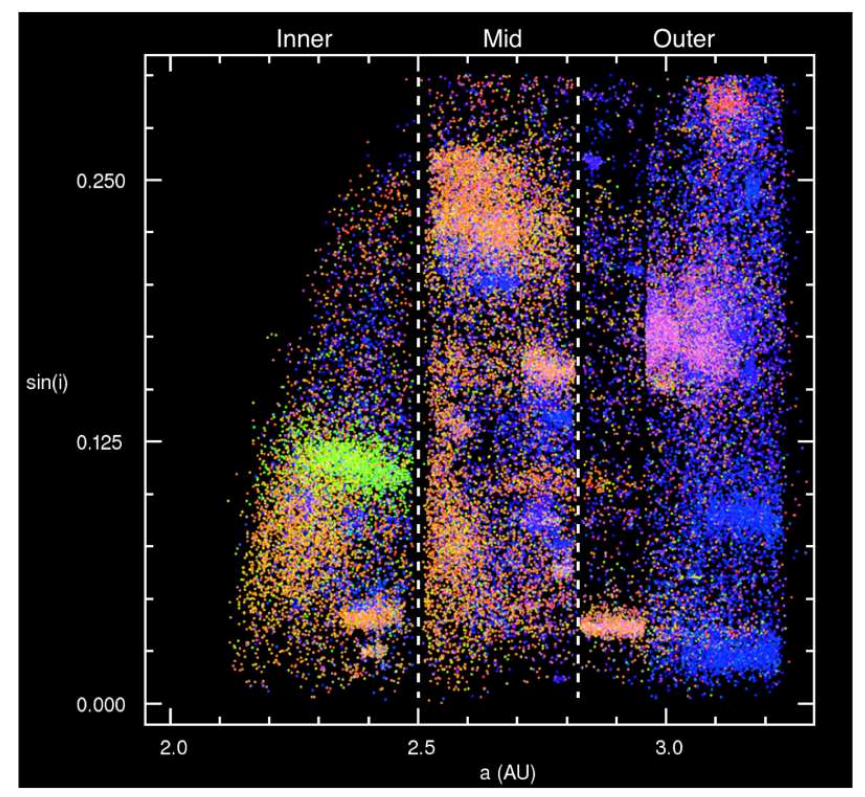

Fig. 4. A plot of the proper $a$ vs. $\sin (i)$ for the same objects as shown in Figure 3. The color of each dot is representative of the object's color measured by SDSS, according to the color scheme defined in Figure 3. The three main regions of the belt, defined by strong Kirkwood gaps, are marked. 

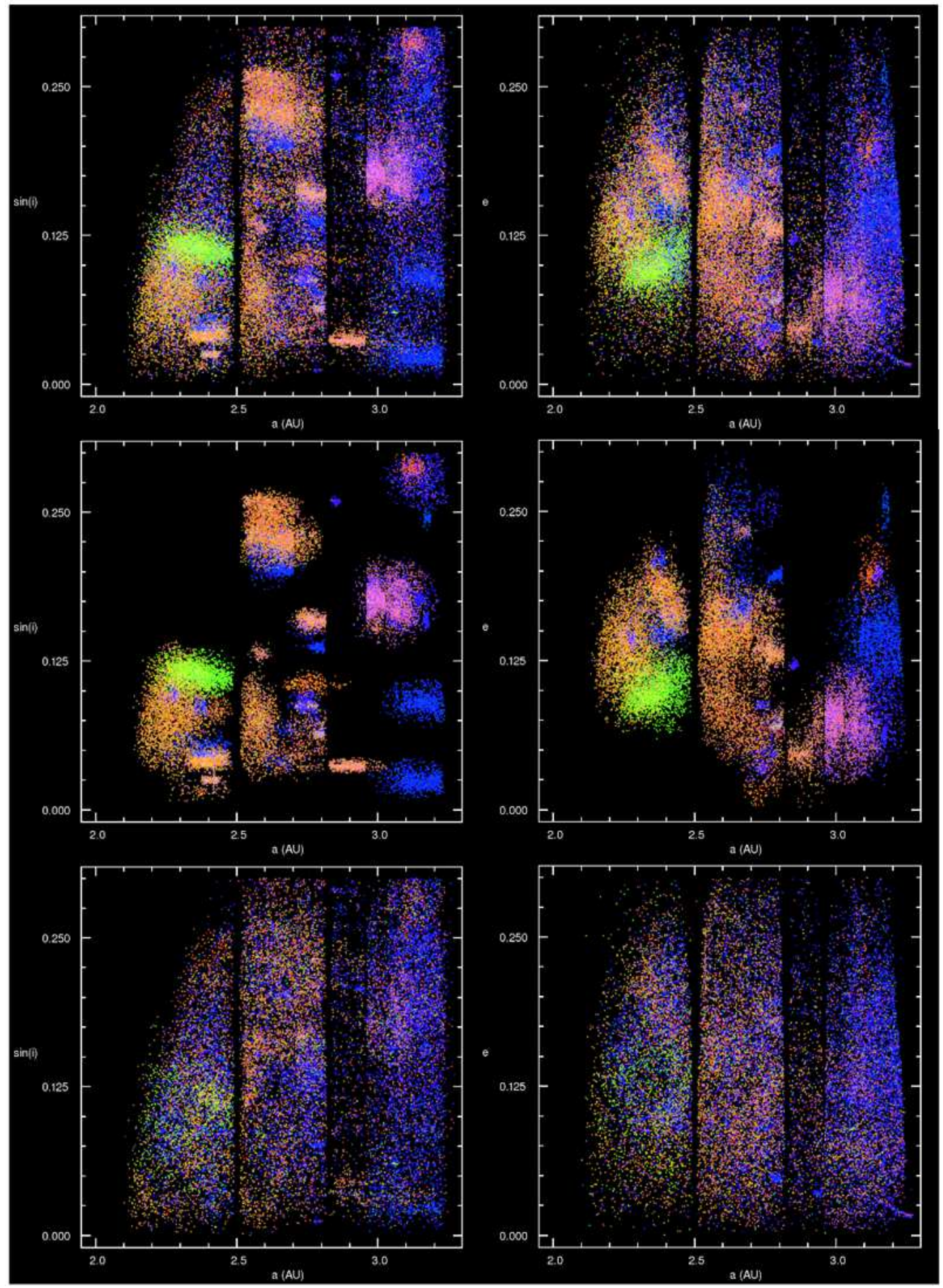

Fig. 5. Illustration of the decomposition of the main-belt asteroid population into families and background objects in proper $a$ vs. $\sin (i)$ (left panels) and proper $a$ vs. $e$ (right panels). The top panels show all (background and family) objects in the data subset. The two middle panels show objects from 37 identified families, and the bottom two panels show the background population. 


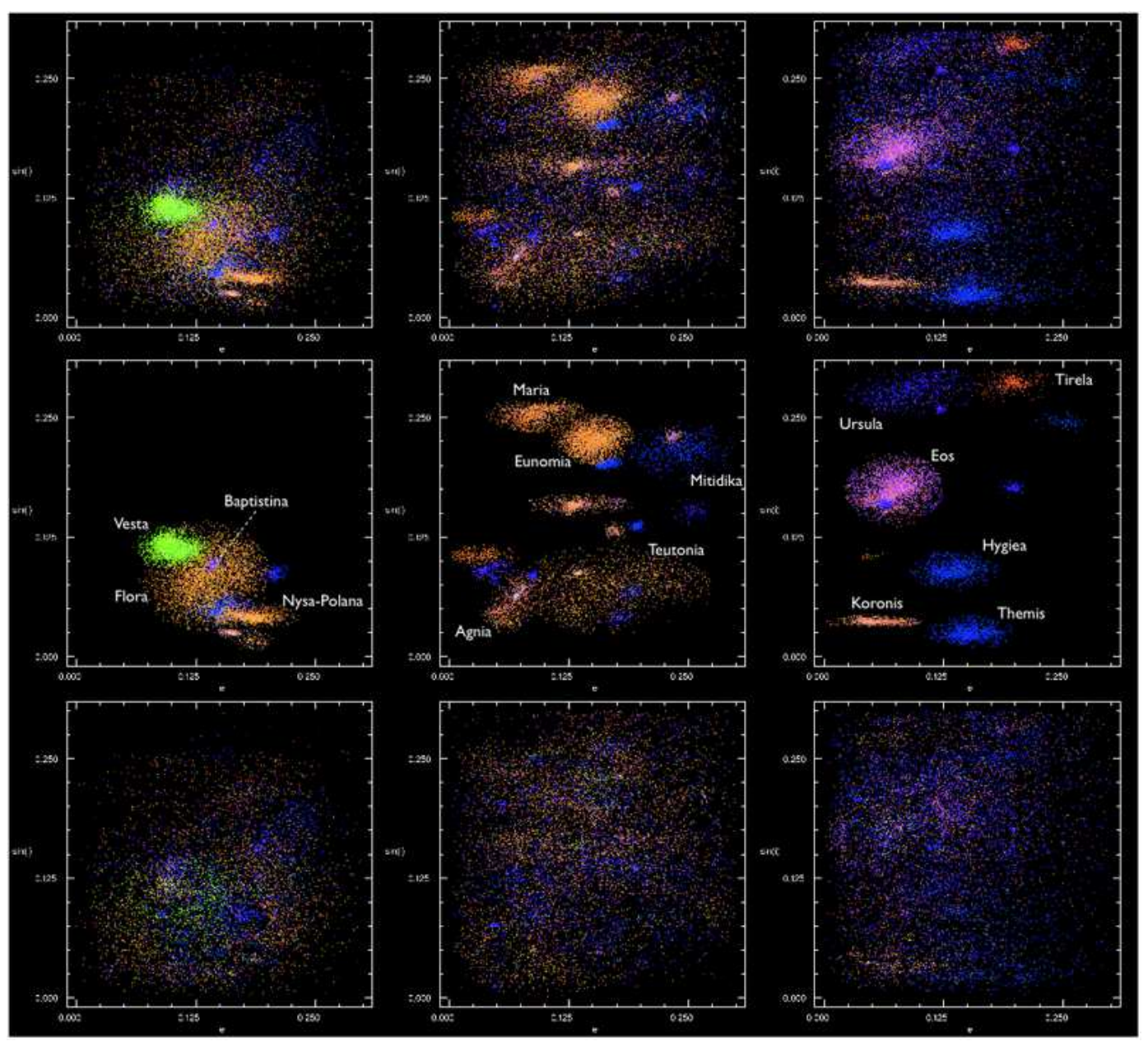

Fig. 6. Analogous to Figures 5, except that the top three panels show the $e$ vs $\sin (i)$ distribution for the three main regions defined by strong Kirkwood gaps ( $a<2.5$ left, $2.5<a<2.82$ middle, $2.82<a<3.5$ right; see Figure 4). The middle row shows family members (with several families of note labeled), and the bottom row shows the background population. For a high-resolution version of this figure with complete labeling, see http://www.astro.washington.edu/ivezic/sdssmoc/sdssmoc.html 


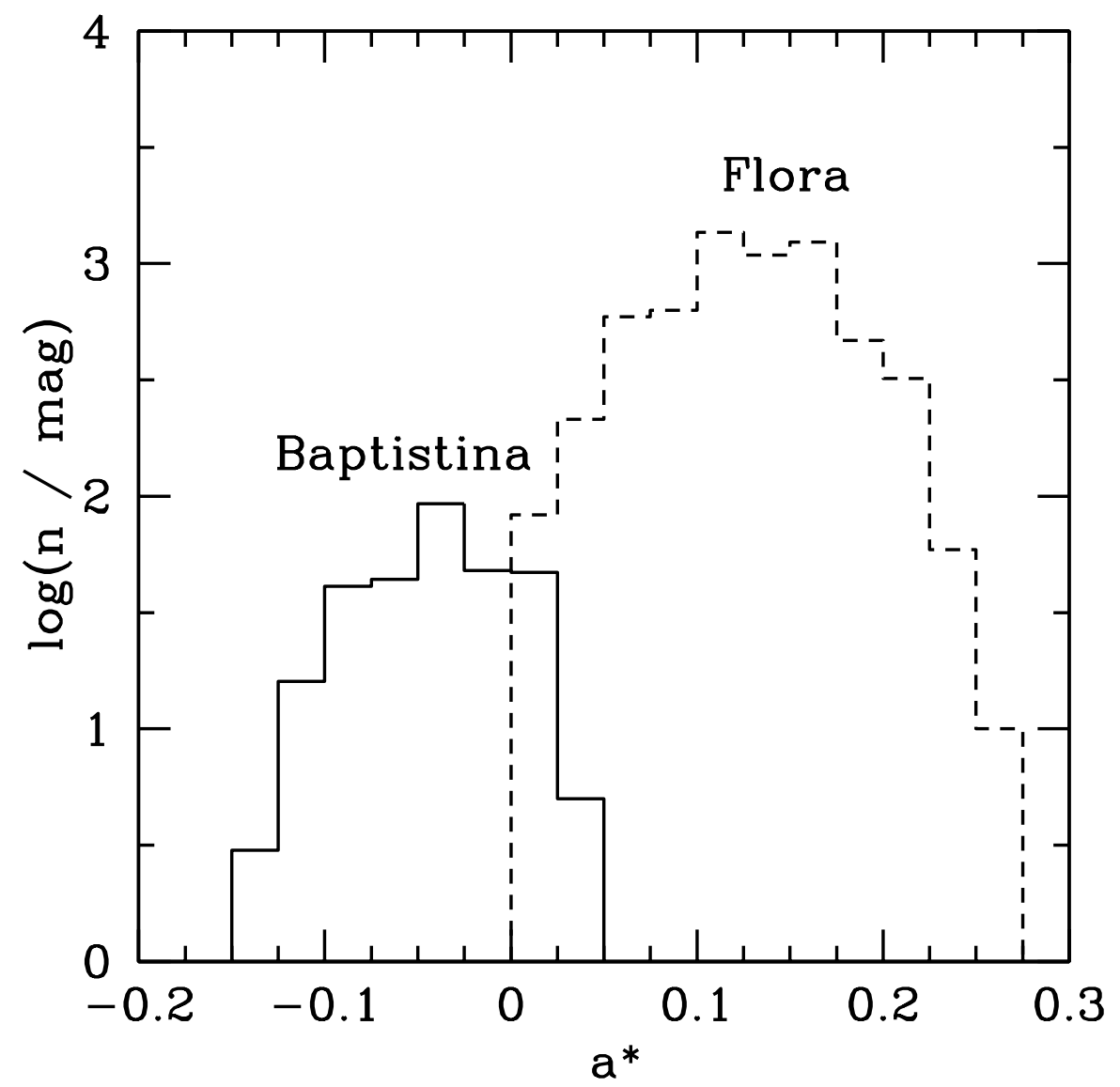

Fig. 7. An illustration of color differences for families with practically identical orbital parameter distributions. The dashed histogram shows the $a^{*}$ color distribution for 6,164 candidate members of the Flora family. The solid histogram shows the $a^{*}$ color distribution for 310 candidate members of the Baptistina family, which is easily separated from the Flora family thanks to the SDSS color information. 

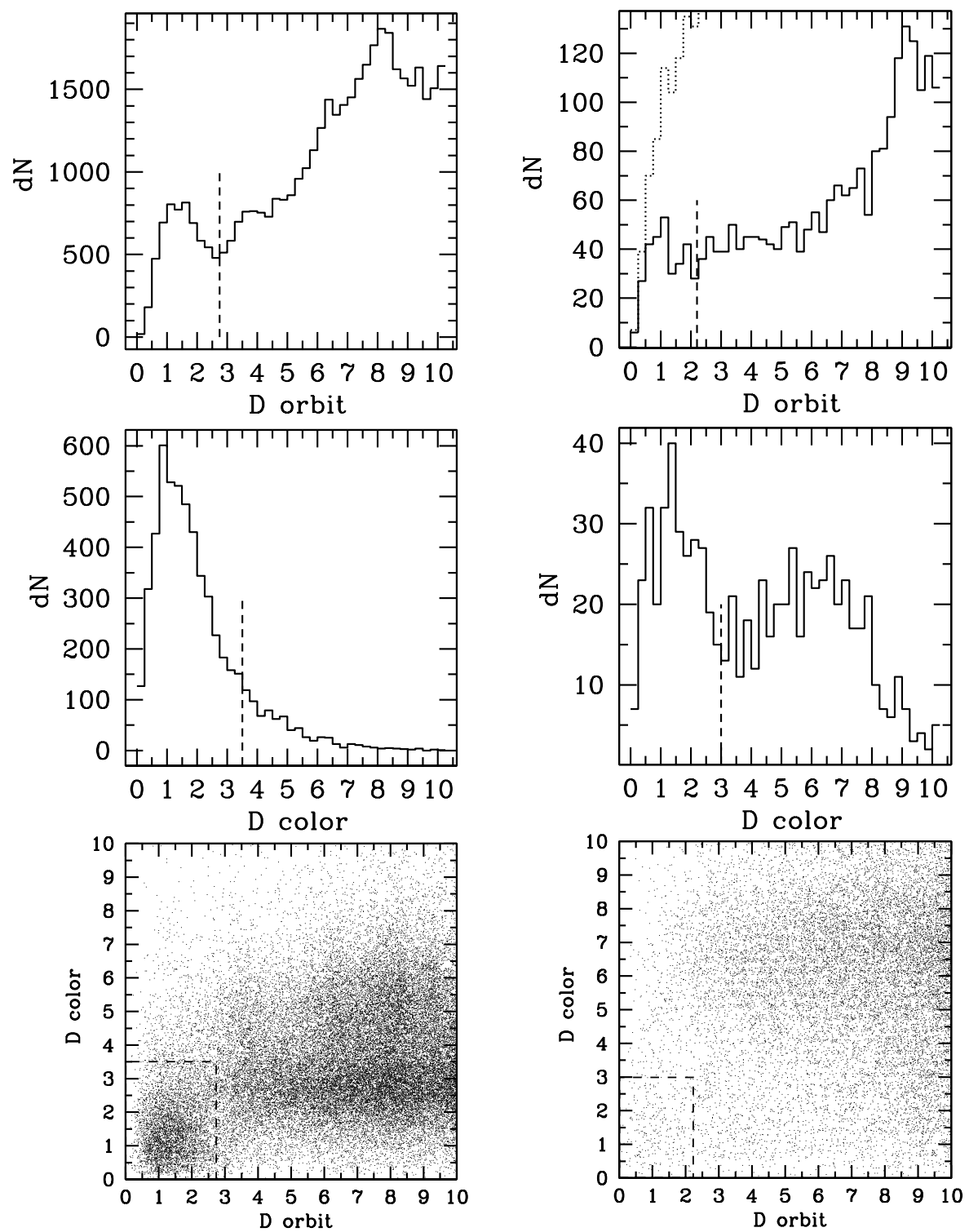

Fig. 8. Top Left: $D_{\text {orbit }}$ histogram for the Vesta family and surrounding objects (defined from the Vesta family centroid). Top Right: Equivalent to top left plot, but for the dynamically buried Baptistina family. The dotted line represents the $D_{\text {orbit }}$ histogram without any color constraints, which climbs smoothly to very high numbers of objects because of the inclusion of Flora family objects. The solid line represents the $D_{\text {orbit }}$ histogram with the color constraints applied, which displays a stronger clustering signature. Vertical dashed line represents $\Delta_{O r b}$ cutoff values selected for these families. Middle Left: $D_{\text {color }}$ histogram for objects that met the $D_{\text {orbit }}$ criteria for the Vesta family. Middle Right: $D_{\text {color }}$ histogram for objects in a preliminary orbital definition of the Baptistina family, showing strong color distinction from the background Flora objects. Vertical dashed line represents $\Delta_{C o l}$ cutoff values selected for these families. Bottom Left and Right: $D_{o r b i t}$ vs. $D_{\text {color }}$ for Vesta and Baptistina families, respectively. Dashed box defines $\Delta_{O r b}$ and $\Delta_{C o l}$ boundaries (objects inside are assigned family membership). 

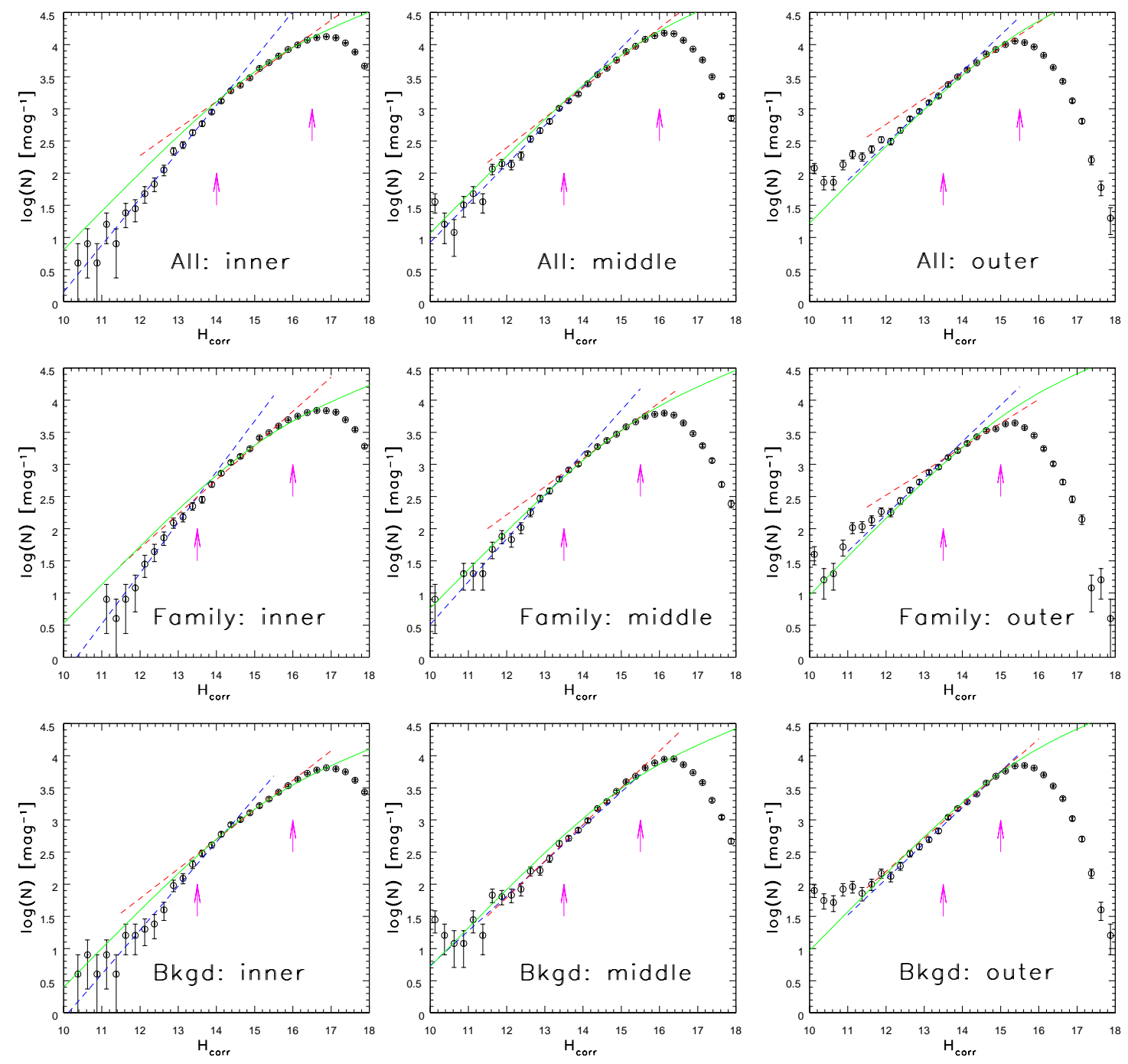

Fig. 9. The differential absolute magnitude distributions corresponding to panels in Figure 6 are shown as symbols with (Poisson) error bars. The solid line shows arbitrarily renormalized best-fit distribution from I01. The two dashed lines show the best-fit "broken" power law: a separate power-law fit for the bright and faint end. In some cases, the two lines are indistinguishable. The best-fit parameters are listed in Table 3. The two arrows show the best-fit break magnitude (left) and the adopted completeness limit (right). 

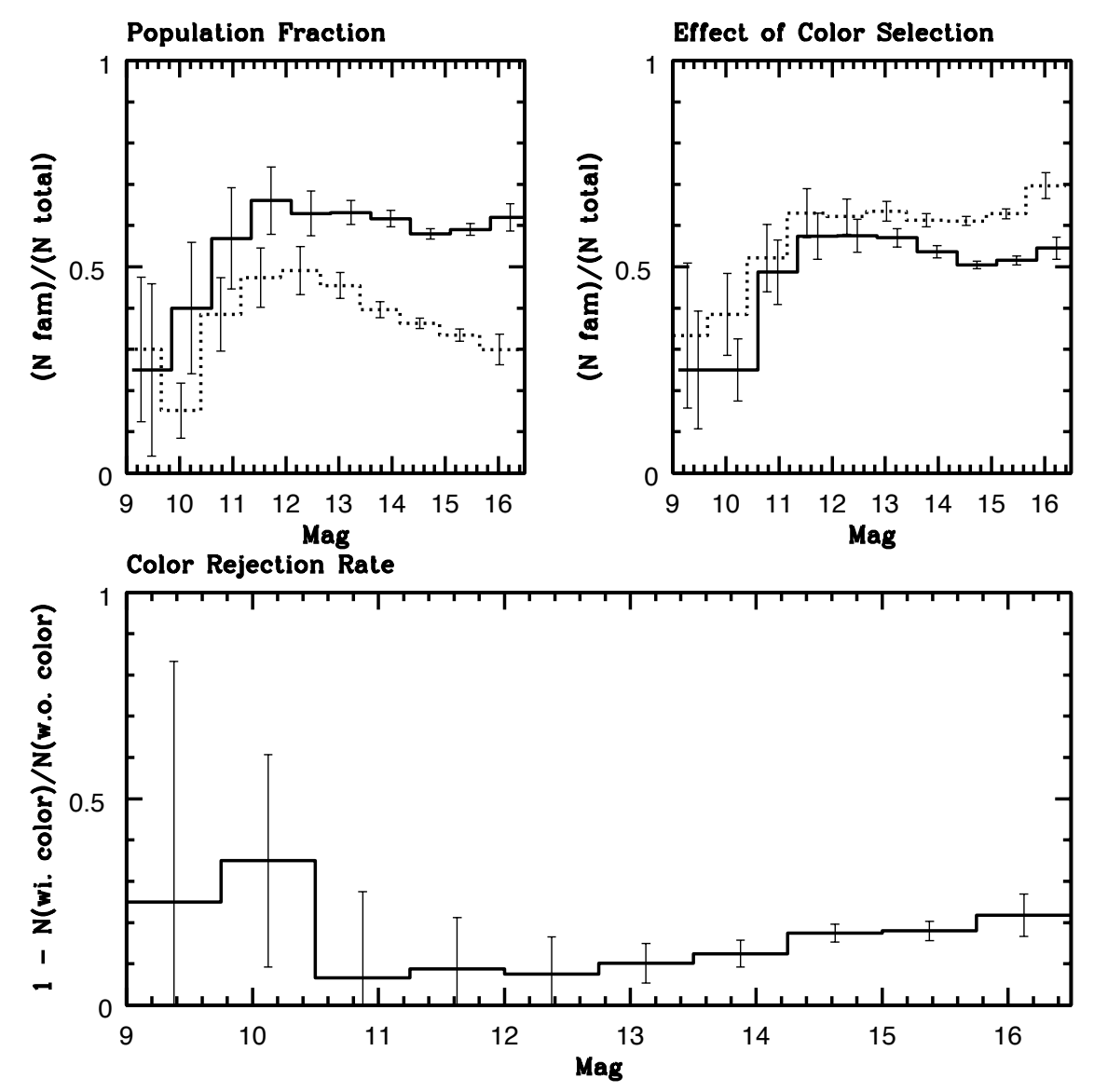

Fig. 10. The family-to-background population ratios and effects of color selection on family populations. The top left panel shows the fraction of objects in families to the total population as a function of $H_{\text {corr }}$ magnitude, with the solid histogram representing red objects $\left(a^{*}>0\right)$ and the dotted histogram representing blue objects $\left(a^{*}<0\right)$. The top right panel compares the fraction of objects in families to the total population as a function of magnitude when colors are used as a constraint on family membership (solid histogram) and when they are not (dotted histogram). The bottom panel shows the rejected fraction due to color constraints as a function of magnitude. 


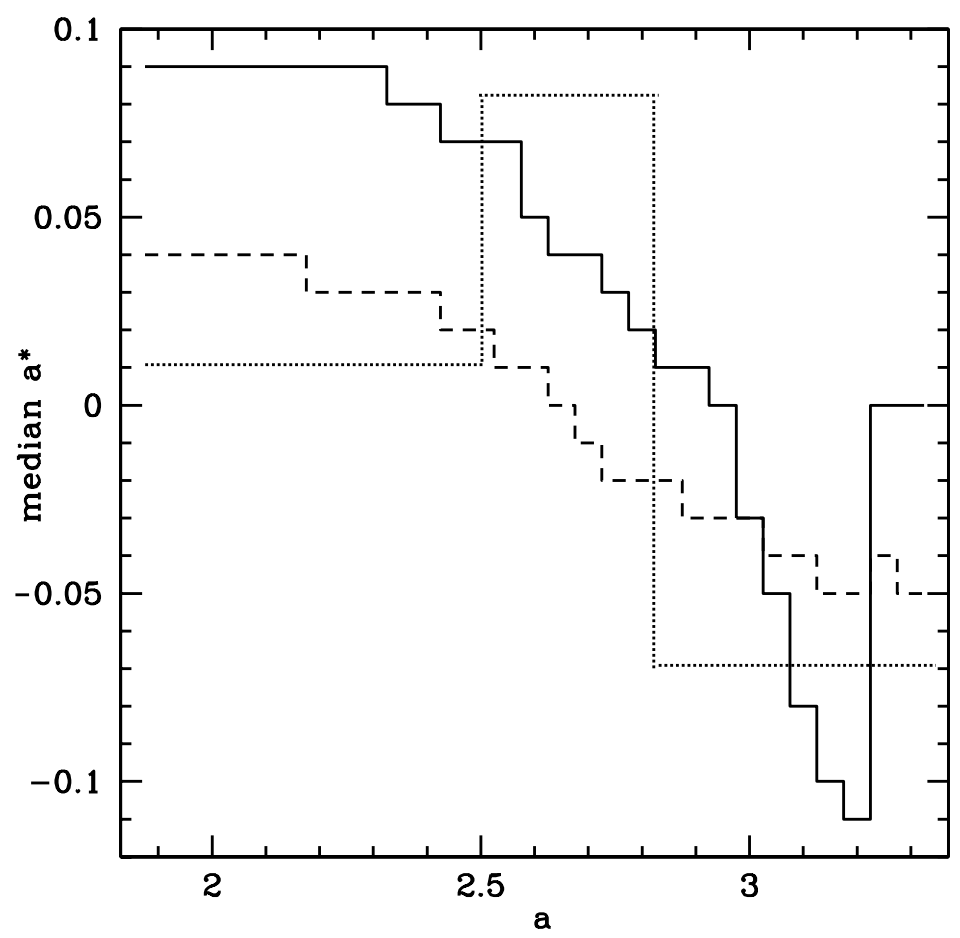

Fig. 11. Median $a^{*}$ color as a function of semi-major axis for several populations. Solid histogram represents the family population, dashed line represents the background population, and the dotted line represents the median of the family $a^{*}$ color centroids (unweighted by family size) for each Main Belt region (inner, mid, and outer). 

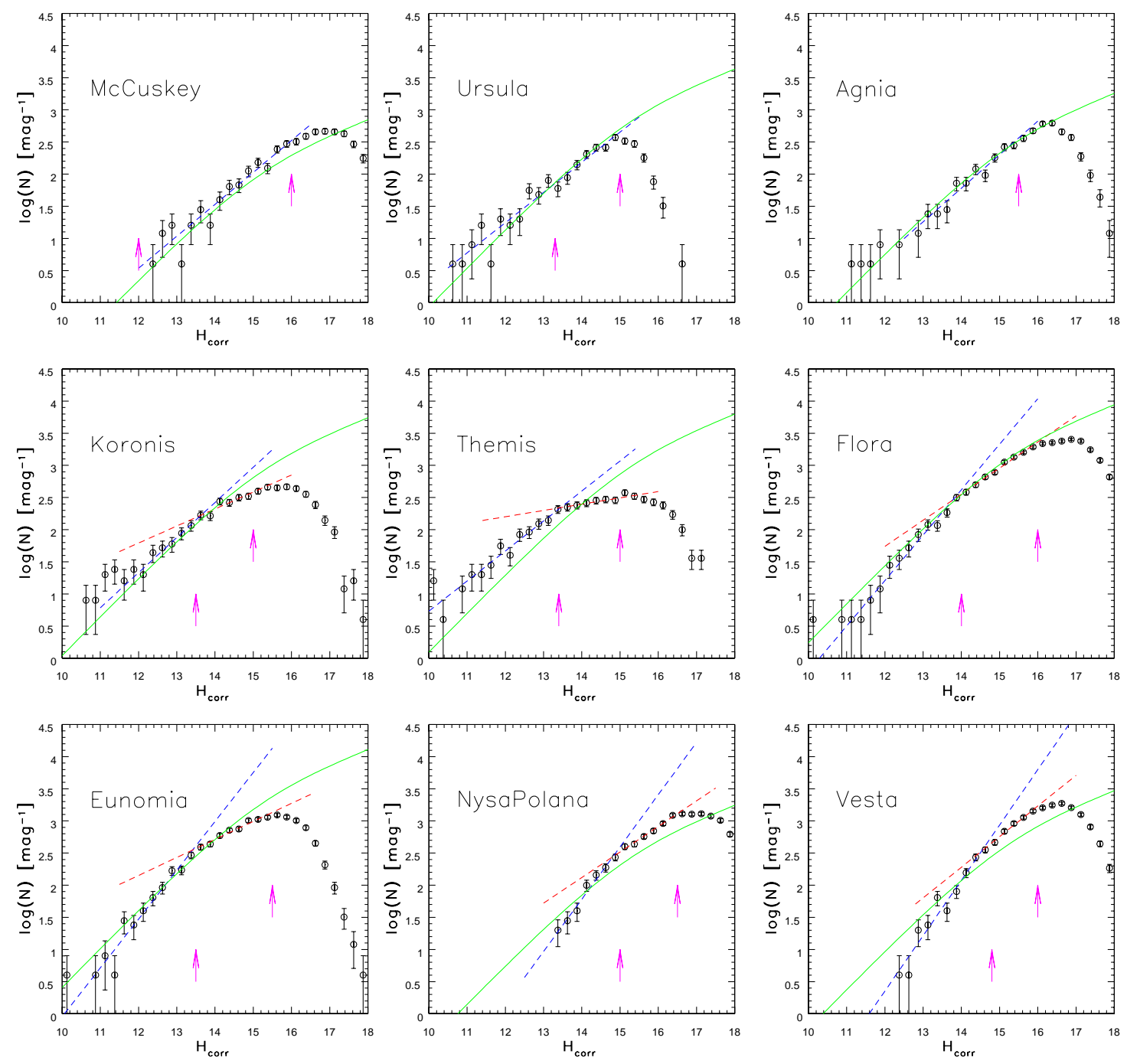

Fig. 12. Analogous to Figure 9, except that the absolute magnitude distributions for selected asteroid families are shown. The first three panels (from top left to bottom right) show examples of families that follow a single power-law magnitude distribution, and the remaining six panels show magnitude distributions for families that require a double power-law fit. The best-fit parameters are listed in Tables 4 and 5 , respectively. 

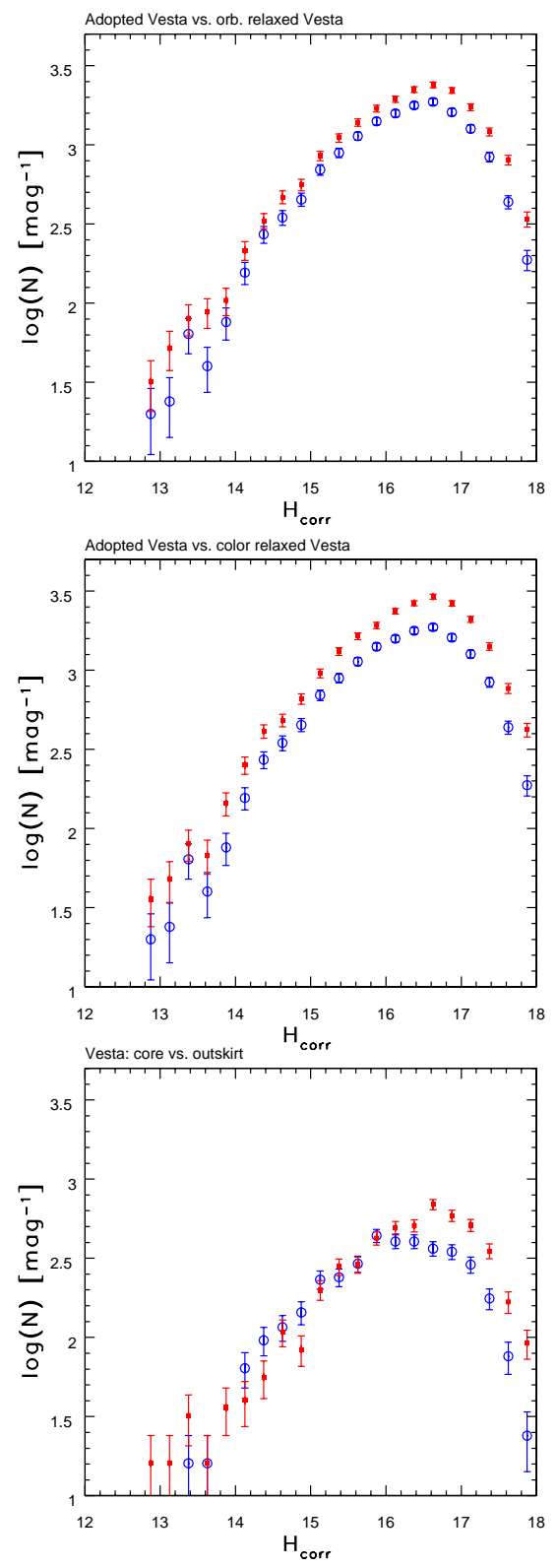

Fig. 13. The dependence of absolute magnitude distribution on family definition for Vesta family. The top panel compares the magnitude distribution obtained for adopted family definition (open circles) to that obtained for a much less constraining cut on orbital elements (closed squares). The middle panel compares the adopted distribution (open circles) to that obtained for a much less constraining color cut (closed squares). In both cases the shapes of the magnitude distributions are similar, with only significant change in the number of selected candidate members. The bottom panel separates the adopted population into the orbital "core" region (open circles) and "outskirt" region (closed squares). Note that the latter distribution is steeper (i.e. the small members are more prevalent in the "outskirt" region). 


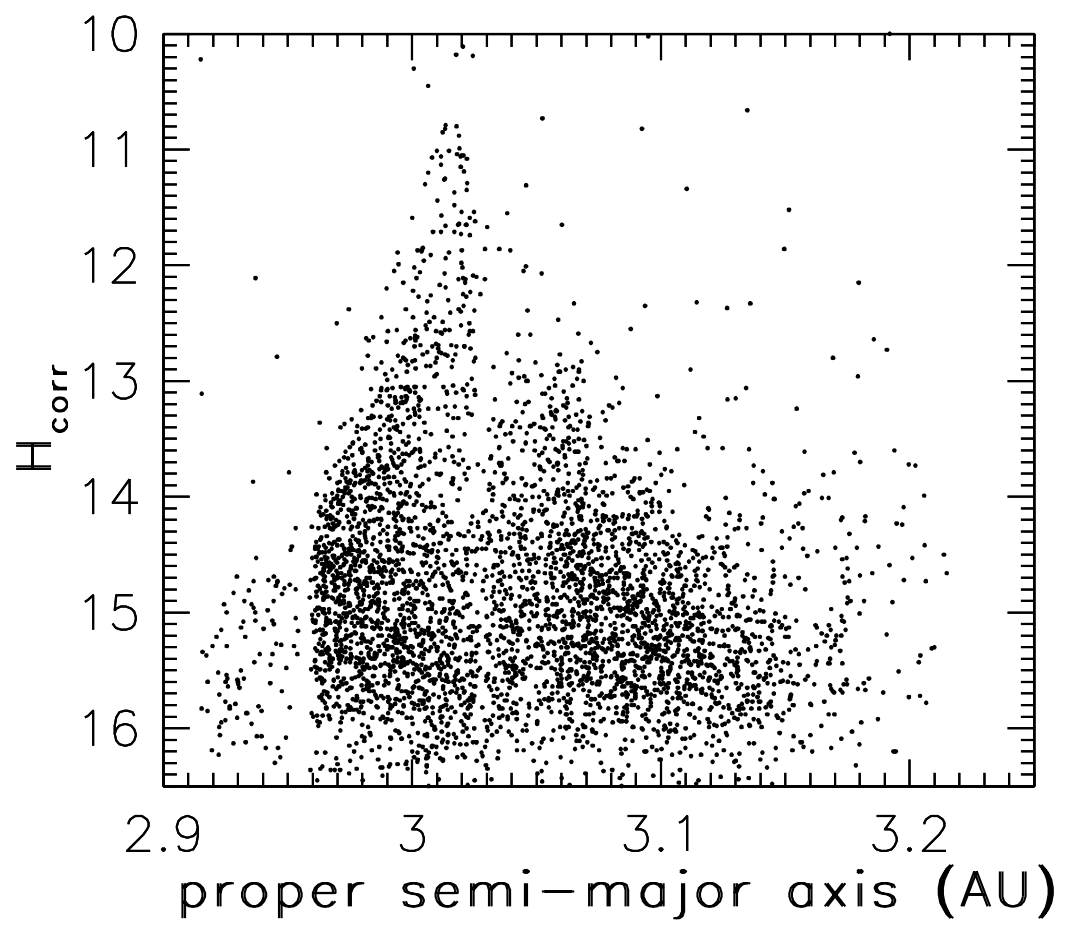

Fig. 14. The correlation between the proper semi-major axis and absolute magnitude for the Eos family. The increased dispersion of the semi-major axis for faint (small) objects is probably caused by the Yarkovsky effect. Note that this family is intersected by several mean motion resonances with Jupiter (e.g. 7:3 at $\left.a_{p} \sim 2.95\right)$ 

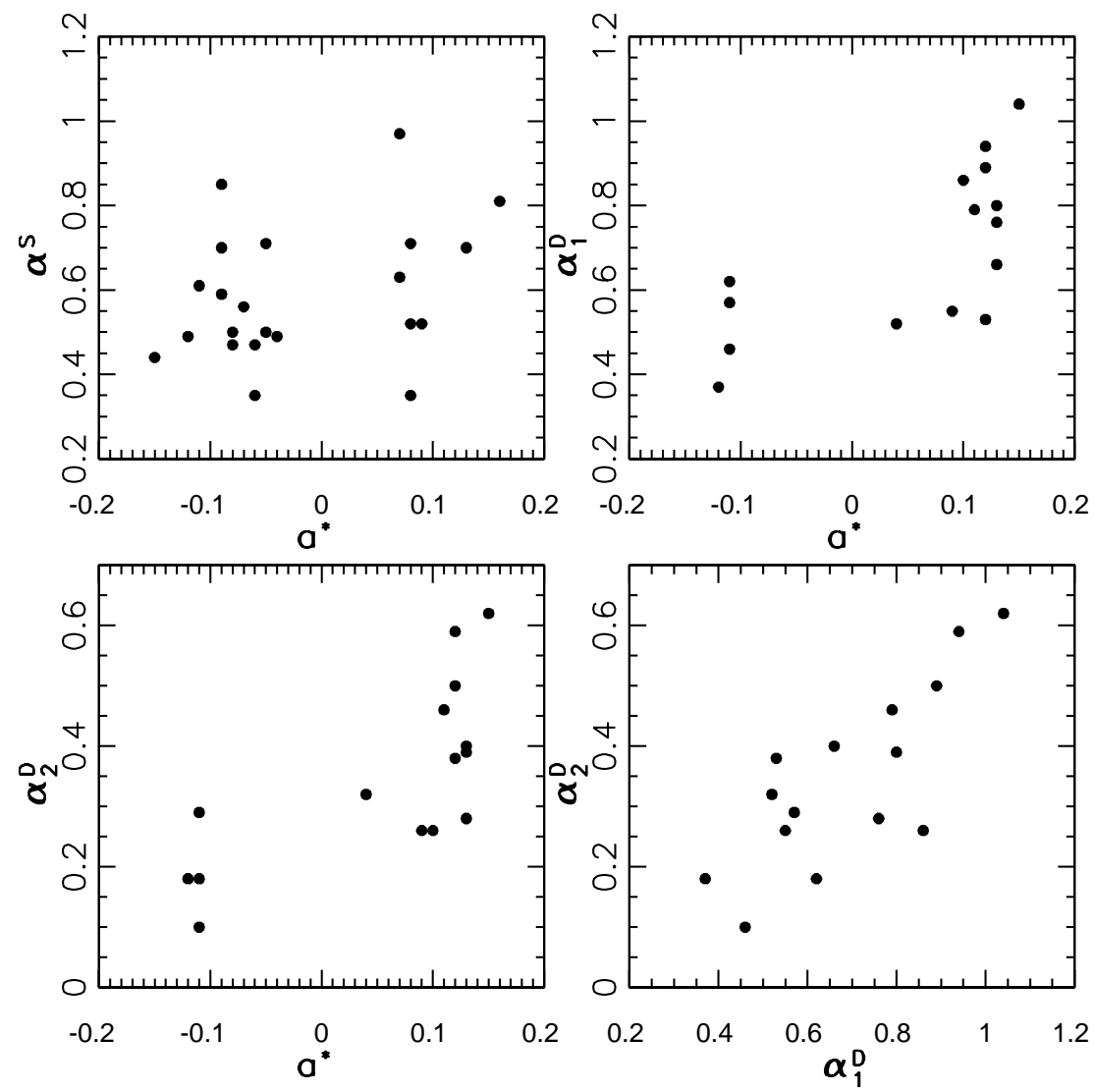

Fig. 15. A summary of relationships between the family median color and parameters of the best-fit power-law magnitude distributions. The top left panel shows the slope of the best-fit power-law as a function of color $a^{*}$ (see text for definition) for 25 families that follow a single power-law magnitude distribution. The top right panel shows the "bright" slope of the best-fit double power-law as a function of color $a^{*}$ for 12 families that follow a double power-law magnitude distribution, and the bottom left panel is analogous plot for the "faint" slope. Note that blue (C type) families have much shallower magnitude distributions than redder families. The bottom right panel demonstrates the strong correlation between the "bright" and "faint" slopes, that seems independent of color. The errors of alpha are dominated by systematics. As per discussion in Section 4.3, we estimate these systematic errors to be about 0.05-0.1. 


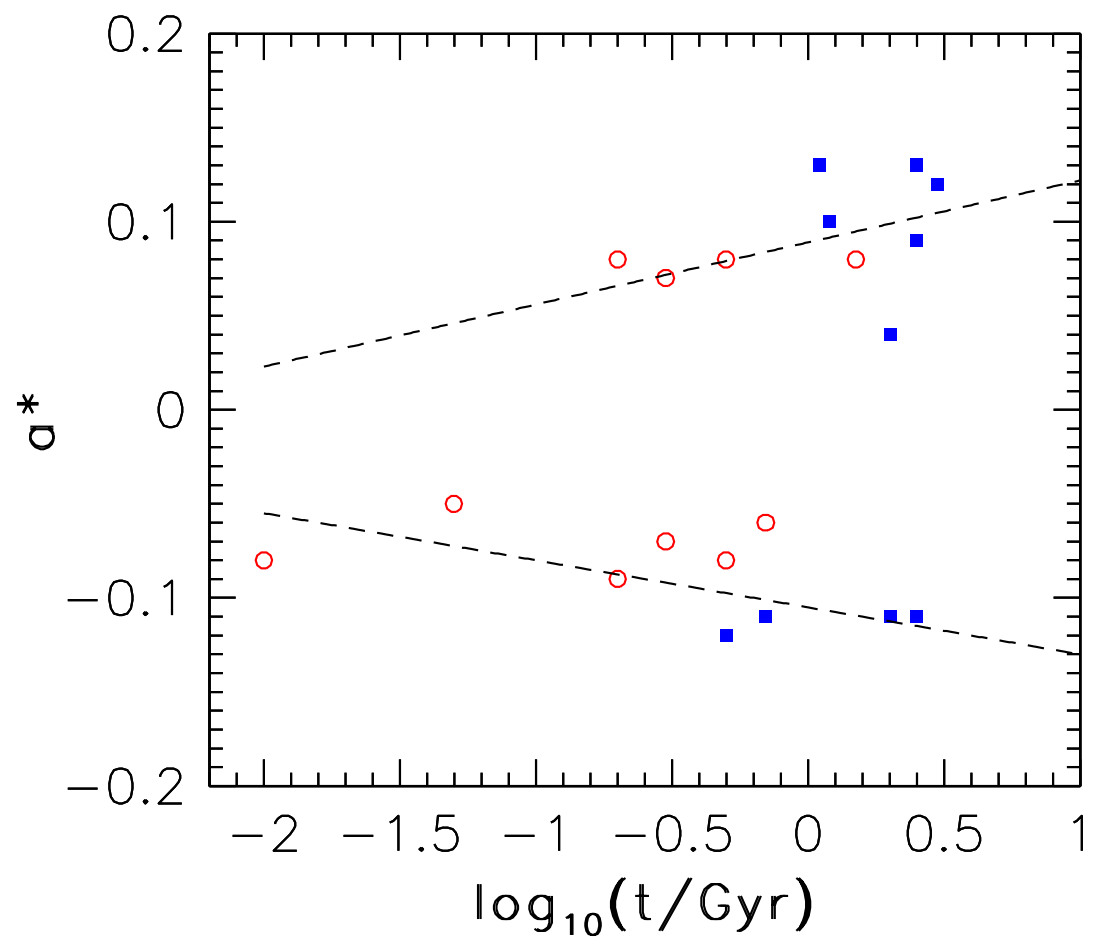

Fig. 16. The correlation between the mean $a^{*}$ color for families defined here and their age taken from N05. Familes whose absolute magnitude distribution can be described by a single power law are shown by circles, and those that require a broken power law as squares. Note that the former are dominated by young families $(\leq 1 \mathrm{Gyr})$, and the latter by old families. The two lines are the best-fit color-age relation from N05, converted using $a^{*}=0.49 P C_{1}-0.16$, where $P C_{1}$ is the first principal color component derived by N05 (note that N05 used several very young familes not discussed here to constrain the slopes of plotted relations). 


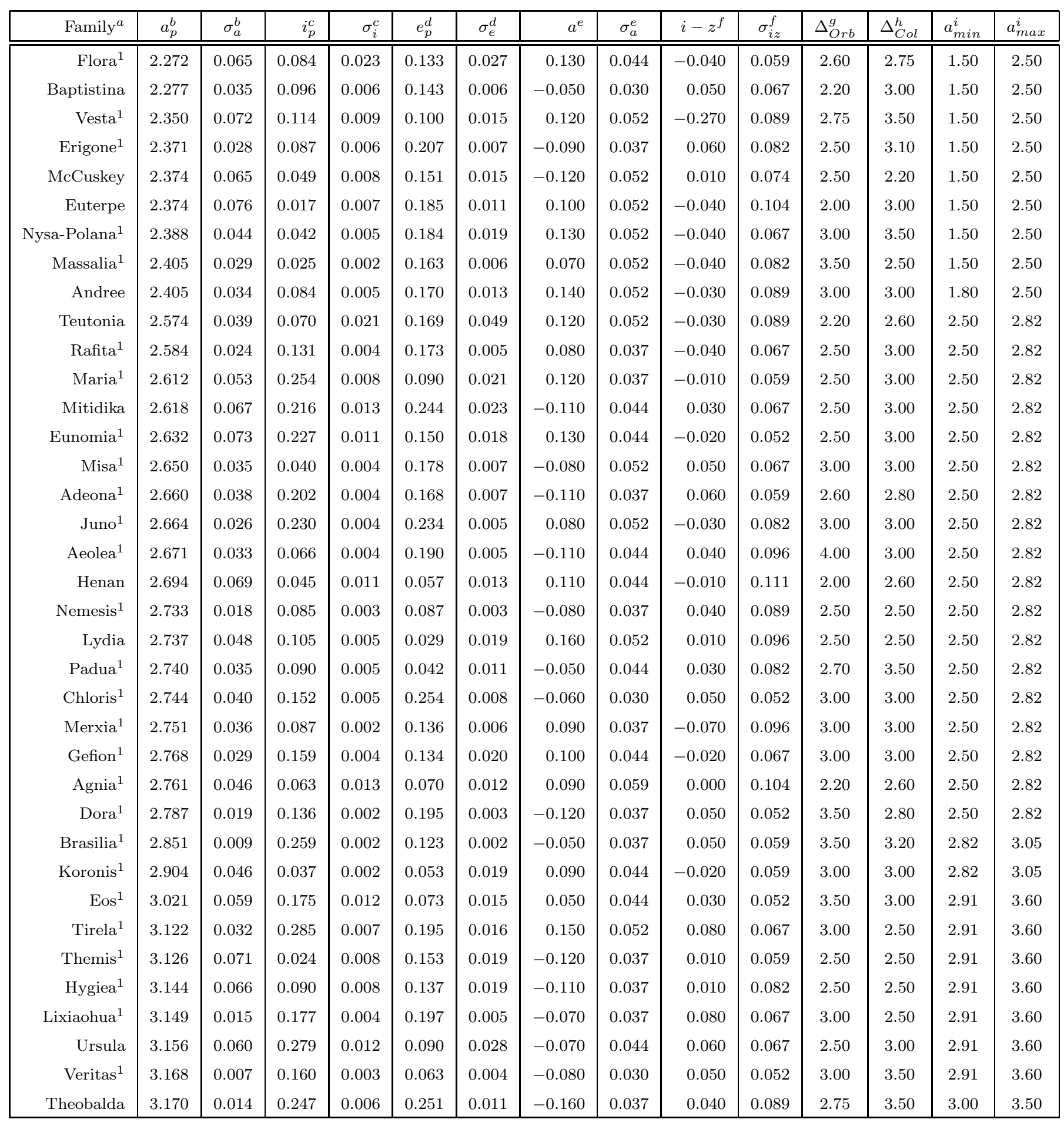

Notes:

${ }^{a}$ the family name (the lowest-numbered member), sorted by semi-major axis

${ }^{b}$ the mean proper semi-major axis (AU) and its gaussian dispersion $(\sigma)$ adopted for this family

${ }^{c}$ the mean sine of proper inclination and its gaussian dispersion adopted for this family

$d$ the mean proper orbital eccentricity and its gaussian dispersion adopted for this family

$e$ the mean $a^{*}$ color (see text for definition) and its gaussian dispersion adopted for this family

$f$ the mean $i-z$ color and its gaussian dispersion adopted for this family

$g$ the maximum deviation in orbital space from the adopted mean values, in units of adopted dispersions (see text)

${ }^{h}$ the maximum deviation in color space from the adopted mean values, in units of adopted dispersions (see text)

${ }^{i}$ the minimum and maximum semi-major axis adopted for this family

${ }^{1}$ family matched to N05

Table 1

Adopted family definitions in the orbital and color space. 


\begin{tabular}{|c|c|c|c|c|c|c|}
\hline Family $^{a}$ & $N^{b}$ & $a_{p}^{c}$ & $i_{p}^{d}$ & $e_{p}^{e}$ & $\mathrm{a}^{f}$ & $i-z^{g}$ \\
\hline Flora & 6164 & 2.28 & 0.08 & 0.13 & 0.13 & -0.05 \\
\hline Baptistina & 310 & 2.28 & 0.10 & 0.14 & -0.04 & 0.03 \\
\hline Vesta & 3793 & 2.35 & 0.11 & 0.10 & 0.12 & -0.32 \\
\hline McCuskey & 1043 & 2.36 & 0.05 & 0.15 & -0.12 & 0.01 \\
\hline Erigone & 307 & 2.37 & 0.09 & 0.21 & -0.09 & 0.05 \\
\hline Euterpe & 387 & 2.38 & 0.02 & 0.18 & 0.09 & -0.03 \\
\hline Nysa-Polana & 2928 & 2.39 & 0.04 & 0.18 & 0.13 & -0.04 \\
\hline Andree & 649 & 2.40 & 0.08 & 0.17 & 0.13 & -0.02 \\
\hline Massalia & 730 & 2.41 & 0.03 & 0.16 & 0.07 & -0.04 \\
\hline Teutonia & 3405 & 2.57 & 0.07 & 0.16 & 0.12 & -0.04 \\
\hline Rafita & 225 & 2.59 & 0.13 & 0.17 & 0.08 & -0.04 \\
\hline Maria & 1315 & 2.61 & 0.25 & 0.09 & 0.12 & -0.02 \\
\hline Mitidika & 698 & 2.61 & 0.22 & 0.24 & -0.11 & 0.03 \\
\hline Eunomia & 2995 & 2.63 & 0.23 & 0.15 & 0.13 & -0.03 \\
\hline Misa & 185 & 2.65 & 0.04 & 0.18 & -0.08 & 0.05 \\
\hline Adeona & 428 & 2.66 & 0.20 & 0.17 & -0.11 & 0.05 \\
\hline Juno & 354 & 2.66 & 0.23 & 0.23 & 0.07 & -0.03 \\
\hline Aeolea & 172 & 2.66 & 0.07 & 0.19 & -0.09 & 0.04 \\
\hline Henan & 624 & 2.67 & 0.04 & 0.06 & 0.11 & -0.02 \\
\hline Nemesis & 129 & 2.73 & 0.09 & 0.09 & -0.09 & 0.02 \\
\hline Lydia & 598 & 2.74 & 0.11 & 0.03 & 0.16 & 0.01 \\
\hline Padua & 442 & 2.75 & 0.09 & 0.04 & -0.05 & 0.05 \\
\hline Merxia & 252 & 2.75 & 0.09 & 0.14 & 0.08 & -0.07 \\
\hline Gefion & 914 & 2.76 & 0.16 & 0.13 & 0.10 & -0.03 \\
\hline Chloris & 121 & 2.76 & 0.15 & 0.25 & -0.06 & 0.05 \\
\hline Agnia & 1106 & 2.76 & 0.06 & 0.07 & 0.08 & -0.01 \\
\hline Dora & 248 & 2.79 & 0.14 & 0.20 & -0.12 & 0.04 \\
\hline Brasilia & 127 & 2.85 & 0.26 & 0.12 & -0.05 & 0.03 \\
\hline Koronis & 1267 & 2.90 & 0.04 & 0.05 & 0.09 & -0.02 \\
\hline Eos & 4367 & 3.04 & 0.18 & 0.07 & 0.04 & 0.03 \\
\hline Tirela & 411 & 3.12 & 0.29 & 0.20 & 0.15 & 0.08 \\
\hline Themis & 1073 & 3.13 & 0.02 & 0.15 & -0.11 & 0.01 \\
\hline Hygiea & 1076 & 3.15 & 0.09 & 0.13 & -0.11 & 0.01 \\
\hline Lixiaohua & 150 & 3.15 & 0.18 & 0.20 & -0.07 & 0.05 \\
\hline Ursula & 644 & 3.15 & 0.28 & 0.09 & -0.06 & 0.06 \\
\hline Veritas & 250 & 3.17 & 0.16 & 0.06 & -0.08 & 0.05 \\
\hline Theobalda & 100 & 3.17 & 0.25 & 0.25 & -0.15 & 0.01 \\
\hline$N A^{h}$ & 90 & 2.87 & 0.11 & 0.05 & 0.17 & 0.04 \\
\hline$N A^{h}$ & 5 & 2.77 & 0.08 & 0.09 & -0.08 & 0.14 \\
\hline$N A^{h}$ & 46 & 2.78 & 0.06 & 0.07 & 0.00 & -0.25 \\
\hline
\end{tabular}

Notes:

${ }^{a}$ the family name (the lowest-numbered member)

$b$ the number of objects in SDSS MOC 4 associated with this family

${ }^{c}$ the median proper semi-major axis (AU)

${ }^{d}$ the median sin of proper inclination

$e$ the median proper orbital eccentricity

$f$ the median $a^{*}$ color (see text for definition)

$g$ the median $i-z$ color

${ }^{h}$ rejected clumps with less than 100 members

Table 2

The median orbital parameters and SDSS colors for detected families and rejected clumps (last three). 


\begin{tabular}{|r|r|c|c|c|c|c|}
\hline$a$ range $^{a}$ & $\mathrm{~N}^{b}$ & $\mathrm{H}_{\min }^{c}$ & $\mathrm{H}_{\max }^{c}$ & $\mathrm{H}_{B}^{d}$ & $\alpha_{1}^{e}$ & $\alpha_{2}^{f}$ \\
\hline \hline $2.00-2.50$ & 30,702 & 11.0 & 16.5 & 14.0 & 0.76 & 0.46 \\
family & 16,309 & 11.0 & 16.0 & 13.5 & 0.79 & 0.53 \\
background & 14,393 & 11.0 & 16.0 & 13.5 & 0.69 & 0.46 \\
\hline $2.50-2.82$ & 32,500 & 11.0 & 16.0 & 13.5 & 0.73 & 0.42 \\
family & 14,261 & 11.0 & 16.0 & 13.5 & 0.67 & 0.44 \\
background & 18,239 & 11.0 & 15.5 & 13.5 & 0.54 & 0.57 \\
\hline $2.82-3.60$ & 24,367 & 12.0 & 15.5 & 13.5 & 0.56 & 0.40 \\
family & 9,547 & 12.0 & 15.0 & 13.5 & 0.57 & 0.37 \\
background & 14,820 & 12.0 & 15.0 & 13.5 & 0.56 & 0.52 \\
\hline
\end{tabular}

Notes:

${ }^{a}$ the range of proper semi-major axis for defining the inner, middle and outer main belt (AU);

${ }^{b}$ the number of objects in each subsample; the first line corresponds to the full sample, and the following two to subsamples classified as families and background, respectively. The total number of objects is 87,569 .

${ }^{c}$ the minimum and maximum $H$ magnitude used in fitting the $H$ distribution

${ }^{d}$ the best-fit "break" $H$ magnitude (see text)

$e$ the "bright" $H$ distribution slope

$f$ the "faint" $H$ distribution slope

Table 3

Best-fit parameters for counts shown in Fig. 9

\begin{tabular}{|c|c|c|c|c|c|}
\hline Family $^{a}$ & $N^{b}$ & $H_{b}^{c}$ & $H_{f}^{d}$ & $\alpha_{S}^{e}$ & $\operatorname{age}^{f}$ \\
\hline Baptistina & 310 & 14.0 & 16.0 & 0.49 & - \\
\hline McCuskey & 1043 & 12.5 & 16.0 & 0.49 & - \\
\hline Erigone & 307 & 14.0 & 16.0 & 0.59 & - \\
\hline Euterpe & 387 & 13.5 & 16.0 & 0.52 & - \\
\hline Andree & 649 & 14.0 & 16.0 & 0.70 & - \\
\hline Massalia & 730 & 14.5 & 16.0 & 0.97 & $0.3 \pm 0.1$ \\
\hline Rafita & 225 & 14.0 & 16.0 & 0.35 & $1.5 \pm 0.5$ \\
\hline Mitidika & 698 & 12.5 & 15.5 & 0.61 & - \\
\hline Misa & 185 & 13.0 & 15.5 & 0.47 & $0.5 \pm 0.2$ \\
\hline Juno & 354 & 14.5 & 16.0 & 0.63 & - \\
\hline Aeolea & 172 & 14.0 & 15.5 & 0.85 & - \\
\hline Nemesis & 129 & 14.0 & 16.0 & 0.70 & $0.2 \pm 0.1$ \\
\hline Lydia & 598 & 14.0 & 16.0 & 0.81 & - \\
\hline Padua & 442 & 14.0 & 16.0 & 0.50 & - \\
\hline Merxia & 252 & 14.5 & 15.5 & 0.71 & $0.5 \pm 0.2$ \\
\hline Chloris & 121 & 13.0 & 15.5 & 0.35 & $0.7 \pm 0.4$ \\
\hline Agnia & 1106 & 13.0 & 15.5 & 0.52 & $0.2 \pm 0.1$ \\
\hline Brasilia & 127 & 13.5 & 15.5 & 0.71 & $0.05 \pm 0.04$ \\
\hline Lixiaohua & 150 & 13.0 & 15.0 & 0.56 & $0.3 \pm 0.2$ \\
\hline Ursula & 644 & 11.0 & 15.0 & 0.47 & - \\
\hline Theobalda & 100 & 13.0 & 15.5 & 0.44 & - \\
\hline Veritas & 250 & 12.0 & 15.0 & 0.50 & $8.3 \pm 0.5 \mathrm{Myr}$ \\
\hline
\end{tabular}

${ }^{a}$ the family name (the lowest-numbered member)

${ }^{b}$ the number of objects in SDSS MOC 4 associated with this family

${ }^{c}$ bright $H$ magnitude limit used for fitting

${ }^{d}$ faint $H$ magnitude limit used for fitting

${ }^{e}$ the best-fit power-law index for $H$ distribution

Table 4

${ }^{e}$ the family age in Gyr (except for Veritas in Myr), taken from Nesvorný et al. (2005), when available

Best-fit $H$ distribution parameters for families described by a single power law. 


\begin{tabular}{|r|r|r|r|r|r|r|r|}
\hline Family $^{a}$ & $N^{b}$ & $H_{b}^{c}$ & $H_{f}^{d}$ & $H_{B}^{e}$ & $\alpha_{1}^{f}$ & $\alpha_{2}^{g}$ & age $^{h}$ \\
\hline \hline Flora & 6164 & 11.0 & 16.0 & 14.0 & 0.66 & 0.40 & $1.1 \pm 0.5$ \\
Vesta & 3793 & 12.5 & 16.0 & 14.8 & 0.89 & 0.50 & - \\
NysaPolana & 2928 & 13.5 & 16.5 & 15.0 & 0.80 & 0.39 & - \\
Teutonia & 3405 & 12.5 & 15.5 & 14.0 & 0.94 & 0.59 & \\
Maria & 1315 & 11.0 & 15.5 & 14.5 & 0.53 & 0.38 & $3.0 \pm 1.0$ \\
Eunomia & 2995 & 11.0 & 15.5 & 13.5 & 0.76 & 0.28 & $2.5 \pm 0.5$ \\
Adeona & 428 & 12.0 & 15.5 & 14.2 & 0.57 & 0.29 & $0.7 \pm 0.5$ \\
Henan & 624 & 14.0 & 16.0 & 15.1 & 0.79 & 0.46 & \\
Gefion & 914 & 12.0 & 15.5 & 14.2 & 0.86 & 0.26 & $1.2 \pm 0.4$ \\
Dora & 248 & 13.0 & 16.0 & 14.8 & 0.37 & 0.18 & $0.5 \pm 0.2$ \\
Koronis & 1267 & 12.0 & 15.0 & 13.5 & 0.55 & 0.26 & $2.5 \pm 1.0$ \\
Eos & 4367 & 10.5 & 15.0 & 13.9 & 0.52 & 0.32 & $2.0 \pm 0.5$ \\
Tirela & 411 & 13.2 & 15.0 & 14.0 & 1.04 & 0.62 & \\
Themis & 1073 & 11.0 & 15.0 & 13.4 & 0.46 & 0.10 & $2.5 \pm 1.0$ \\
Hygiea & 1076 & 12.7 & 15.0 & 14.4 & 0.62 & 0.18 & $2.0 \pm 1.0$ \\
\hline
\end{tabular}

Notes:

$a$ the family name (the lowest-numbered member)

$b$ the number of objects in SDSS MOC 4 associated with this family

${ }^{c}$ bright $H$ magnitude limit used for fitting

${ }^{d}$ faint $H$ magnitude limit used for fitting

$e$ the "break" $H$ magnitude used for fitting

$f$ the best-fit power-law index for $H$ distribution at the bright end

$g$ the best-fit power-law index for $H$ distribution at the faint end

$h$ the family age in Gyr, taken from Nesvorný et al. (2005), when available

Table 5

Best-fit $H$ distribution parameters for families described by a "broken" power law. 